\title{
El impacto de la crisis de seguridad contemporánea inducida políticamente en el comportamiento de los turistas: la percepción de la población joven serbia
}

\author{
The Impact of Contemporary Politically Induced Security Crisis on Tourist \\ Behaviour: The perception of the Serbian Younger Adult Population
}

\author{
Stefan Denda, Jasna Micić y Aleksandra Terzić
}

\section{Palabras clave \\ Actitudes de turistas \\ - Elección de destino turístico \\ - Jóvenes \\ - Países mediterráneos \\ - Primavera Árabe \\ - Riesgo de seguridad \\ - Serbia \\ - Violencia política e impactos turísticos}

\section{Key words}

Tourists' Attitudes

- Tourism Destination

Choice

- Young Adult and

Young Middle-Aged

- Mediterranean

Countries

- Arab Uprisings

- Security Risks

- Serbian

- Political Violence and

Tourist Impacts

\begin{abstract}
Resumen
La investigación tiene por objetivo determinar cómo los turistas perciben la crisis de seguridad en la región MENA y en qué medida afecta al proceso de decisión de viaje. Se realizó entre los residentes jóvenes y de mediana edad serbios. La encuesta hizo un seguimiento de las características demográficas y socioeconómicas de los encuestados, sus hábitos de viaje y actitudes hacia la situación de seguridad en el Mediterráneo. El público objetivo es muy consciente de la situación de seguridad. Las pruebas estadísticas no paramétricas revelaron las diferencias de género en la percepción del riesgo. Aunque la reubicación de la demanda turística de Serbia se ha producido como consecuencia de la crisis, la investigación muestra que la situación de seguridad no es un factor decisivo en la selección del destino.
\end{abstract}

\section{Abstract}

The study examines how tourists perceive the security crisis in the MENA region and to what extent it affects their travel decision process. It is based on a survey conducted among Serbian young adult and younger middle-aged residents. The survey tracked the demographic and socioeconomic characteristics of the respondents, their travel habits and attitudes towards the security situation in the Mediterranean. The research shows that the target population is well-aware of the current security situation. The non-parametric statistical tests conducted reveal differences by gender in risk perception. Even though a relocation of tourism demand in Serbia has occurred because of the security crisis, the study findings indicate that the security situation is not a decisive factor in destination selection.

\section{Cómo citar}

Denda, Stefan; Micić, Jasna y Terzić, Aleksandra (2021). «El impacto de la crisis de seguridad contemporánea inducida políticamente en el comportamiento de los turistas: la percepción de la población joven serbia». Revista Española de Investigaciones Sociológicas, 174: 69-88. (http://dx.doi. org/10.5477/cis/reis.174.69)

La versión en inglés de este artículo puede consultarse en http://reis.cis.es

Stefan Denda: Geografski Institut Jovan Cvijic Srpska Akademija Nauka i Umetnosti (Serbia) | s.denda@gi.sanu.ac.rs Jasna Micić: Geografski Institut Jovan Cvijic Srpska Akademija Nauka i Umetnosti (Serbia) | j.micic@gi.sanu.ac.rs Aleksandra Terzić: Geografski Institut Jovan Cvijic Srpska Akademija Nauka i Umetnosti (Serbia) | a.terzic@gi.sanu.ac.rs 


\section{INTRODUCCIÓN}

El turismo es una industria que ha estado en constante crecimiento durante las últimas décadas (Middleton et al., 2009). En consecuencia, se ha convertido en un recurso importante de ingresos para muchos países de todo el mundo. A pesar de esta tendencia positiva, también se registraron disminuciones: 1967-1968, 1973-1974 (recesión económica), a mediados de los años ochenta (el accidente de Chernóbil, bombardeo de Libia), a principios de los años noventa (la guerra del Golfo y las guerras yugoslavas), década de 2000 (ataques terroristas en los Estados Unidos, Madrid, Londres y Bali; la Guerra de Afganistán y la Guerra de Irak; el SARS, el tsunami asiático y la ceniza islandesa), y la crisis financiera global de 2008-2009 (Vanhove, 2011).

Durante este tiempo, el turismo ha cambiado tanto en términos cuantitativos como cualitativos, por esto, los factores que influyen en el comportamiento de los turistas se volvieron aún más complejos. Existen muchas teorías de motivación turística (Cohen, 1972; Crompton, 1979; Iso-Ahola, 1982, 1983; Mayo y Jarvis, 1981; Plog, 1974) que intentaron sistematizar el caótico proceso de toma de decisiones de viaje. Según Lim (1999), los ingresos, los costos de transporte y los precios del turismo son las variables más utilizadas en las investigaciones empíricas de la demanda turística. A pesar de que se sabe que ciertos eventos tienen un impacto en la sensación de seguridad percibida a nivel global y regional (por ejemplo, terrorismo, desastres naturales, migraciones, enfermedades contagiosas, etc.) (Kôvári y Zimányi, 2011), la relación entre la demanda turística y la seguridad y protección en los destinos turísticos ha percibido una atención limitada tanto por parte de los investigadores como por parte de los responsables políticos (Fourie, Roselló-Nadal y Santana-Gallego, 2020; Ghaderi, Saboori y Khoshkam, 2017). El ataque terrorista contra las Torres Gemelas en Nueva York (2001) cambió la percepción potencial de los turistas sobre la seguridad global, por lo que los viajeros ahora tienen en cuenta esta relación (Lepp y Gibson, 2003).

Desde la década de los noventa, se han registrado ataques terroristas contra viajeros en los destinos turísticos con una población islámica mayoritaria (por ejemplo, Egipto, Túnez y Turquía). Sin embargo, en los últimos años, los turistas en los destinos occidentales (Francia, Alemania, España y Bélgica) también se han convertido en objetivos de ataque. Este fenómeno está asociado con disturbios políticos en los países del Oriente Medio y África del Norte, conocidos como la Primavera Árabe. Desde finales de 2010, estas conflictividades han tenido un gran impacto político y socioeconómico en el Mediterráneo. Como consecuencia directa de esta inestabilidad, Europa ha sufrido la peor crisis migratoria y de seguridad desde la Segunda Guerra Mundial. Además, todos estos eventos han tenido una gran repercusión en el desarrollo de la industria del turismo, especialmente en la región del Medio Oriente y el norte de África (MENA).

Como declararon Mansfeld y Winckler (2015), la crisis de la Primavera Árabe en el turismo ha sido diferente en comparación con otras debido a su duración y a la naturaleza de los nuevos regímenes. Aunque Avraham (2015) reveló los efectos negativos de las crisis mencionadas en la industria del turismo en la región MENA, aún faltan investigaciones empíricas que expliquen los efectos de esta revolución (Tomazos, 2017; Theocharous et al., 2018).

Teniendo en cuenta que la relación entre la seguridad y protección y la demanda del turismo no ha sido bien investigada, este estudio tiene como objetivo llenar el vacío visible en la literatura social y turística actual. El impacto de la crisis de la Primavera Árabe en la demanda turística está en el centro de investigación debido a los efectos globales y a la duración del problema, junto con la impre- 
sión general de la inseguridad en la región MENA, incluso cuando no hay una guerra oficial (Butler y Suntikul, 2013). La pregunta de investigación que intentamos responder es si esta crisis y los eventos que la acompañaron afectaron, y en qué medida, la demanda turística hacia la región MENA.

\section{La Primavera Árabe como UN DESAFÍO DE SEGURIDAD CONTEMPORÁNEO}

Los levantamientos árabes comenzaron el 17 de diciembre de 2010, cuando el vendedor ambulante Mohammed Bouazizi se prendió fuego a sí mismo después de la confiscación de sus bienes por la policía en Túnez (Ansani y Daniele, 2012). Este evento fue conocido como la Revolución del Jazmín. Desde enero hasta noviembre de 2011, el levantamiento árabe fue seguido por una serie de acontecimientos masivos y violentos en los países de la región MENA (Túnez, Egipto, Libia, Marruecos, Yemen, Jordania, Bahréin, Siria y otros). La «calle árabe» y la «ciudad árabe» (la plaza de la Liberación o plaza Tahrir en El Cairo, la plaza de los Mártires en Trípoli, etc.) fueron los lugares de la «ocupación revolucionaria» (Ardıç, 2012). A medida que la juventud urbana conformaba una gran parte de la población, jugaron un papel importante en la revolución. Esta generación (15 a 29 años) era menos religiosa y, por lo tanto, menos partidaria del islam político. Por otro lado, eran más educados, pero muchos de ellos estaban desempleados (representando el $25 \%$ del total de la población desempleada en Túnez y Egipto; casi el 50\% eran mujeres). Su insatisfacción con la situación política y económica fue enorme (Hoffman y Jamal, 2012). La juventud urbana utilizó las tecnologías de la información y la comunicación como el instrumento principal de las reuniones masivas y la propagación del lema (Costello, Jenkins y Aly, 2015).
Los disturbios fueron apoyados por factores externos, como la Unión Europea, EE. UU., Turquía y la OTAN, debido a sus intereses geoestratégicos en la región, especialmente después del 11 de septiembre de 2001.

Al mismo tiempo, el papel de los turcos en este evento fue específico por muchas razones. Para los Estados islámicos, Turquía fue un ejemplo de capitalismo e islam trabajando juntos (Yılmaz, 2012). Además, para Turquía, los vecinos del Medio Oriente eran la puerta de entrada al mundo árabe (económica y políticamente) (Öniş, 2014). Finalmente, este país realizó una auditoría de las prioridades estratégicas y se comprometió a cooperar con Rusia para resolver el conflicto sirio. Debido a esto, la cooperación con los socios occidentales (EE. UU. y UE) disminuyó después del fallido golpe de estado de Turquía de 2016 (D’Alema, 2017).

Todas estas presiones fomentaron cambios políticos en estos países. En Túnez y Egipto, se estableció un nuevo orden político; Marruecos y Jordania hicieron cambios constitucionales, mientras que un nuevo presidente fue elegido en Jordania. Al mismo tiempo, Libia fue completamente fragmentada, mientras que Siria sufrió una guerra civil que está todavía en curso (Salih, 2013). Estos desarrollos llevaron al fortalecimiento del islamismo, la aparición de movimientos políticos (como la Hermandad Musulmana y el Partido Al-Nour en Egipto) y grupos militantes (por ejemplo, el Estado Islámico de Irak y Siria, Ansar al-Sharia (en castellano: Partidarios de la Sharia) en Libia, El Frente Al-Nusra en Siria) (Dalmasso y Cavatorta, 2013).

Estos conflictos causaron migraciones masivas hacia Europa. Según los informes de la agencia, se identifican cuatro mayores (rutas del Mediterráneo central, occidental y oriental, y los Balcanes occidentales), y tres rutas adicionales (ruta circular de Albania a Grecia, las fronteras orientales y la ruta de África occidental). Teniendo en cuenta esta clasificación de rutas, el creciente número 
de migrantes en Grecia (Ruta del Este) tuvo un efecto directo sobre la Ruta de los Balcanes Occidentales (FRONTEX, 2012-2016). Los migrantes intentaron continuar su viaje a Hungría y Croacia a través de Macedonia del Norte y Serbia. Esta ruta se hizo popular en 2012 debido al Acuerdo de Schengen, que abolió los visados para los países de la antigua Yugoslavia y Albania. Desde 2013, el número de cruces fronterizos ha crecido constantemente, llegando a 764.000 en 2015 (14-16 veces más que en 2014) (FRONTEX, 2012-2016).

\section{El impacto de Los Riesgos DE SEGURIDAD EN EL TURISMO del MediterRáneo}

A nivel mundial, los conflictos tuvieron un impacto enorme en todos los aspectos de las relaciones internacionales, incluido el turismo. Más de un tercio de la facturación europea turística se logró en la subregión sur/mediterránea (18-19\% de las llegadas mundiales).
Estos países (Francia, España, Italia, Turquía, Grecia y Croacia) tuvieron tasas de crecimiento anual con un promedio variable de las llegadas del $2 \%$ al 23\%. Individualmente, España y Grecia fueron testigos del mayor crecimiento (2014) y Francia de una menor disminución de visitantes internacionales (2016) debido a incidentes de seguridad en París y Niza (UNWTO, 2011-2017). En 2011 y 2015, el número de llegadas a la región de África disminuyó (en un 3\%) debido a los desafíos geopolíticos, de seguridad y económicos.

Estos problemas tuvieron un gran impacto en la parte norteafricana del Mediterráneo (una disminución del 6-12\%), especialmente en Túnez (-31\% en 2011) debido a los cambios políticos (UNWTO, 2011-2017) (ver tabla 1). Además, en la región del Medio Oriente, se registró un crecimiento negativo (-8\%) debido a los riesgos de seguridad en 2011. A nivel de destino, Egipto experimentó la mayor turbulencia (-31\% en 2011 y 2014 ; $-42 \%$ en 2016) debido a varios incidentes (tensiones políticas, el golpe militar, la guerra civil siria, etc.) (UNWTO, 2011-2017).

TABLA 1. Llegadas de turistas internacionales (2010-2016) en los países seleccionados (en millones)

\begin{tabular}{lccccccc}
\hline País & $\mathbf{2 0 1 0}$ & $\mathbf{2 0 1 1}$ & $\mathbf{2 0 1 2}$ & $\mathbf{2 0 1 3}$ & $\mathbf{2 0 1 4}$ & $\mathbf{2 0 1 5}$ & $\mathbf{2 0 1 6}$ \\
\hline España & 52,7 & 56,7 & 57,7 & 60,6 & 65,0 & 68,2 & 75,6 \\
Francia & $\mathbf{7 6 , 8}$ & $\mathbf{7 9 , 6}$ & $\mathbf{8 3 , 0}$ & $\mathbf{8 3 , 6}$ & $\mathbf{8 3 , 7}$ & $\mathbf{8 3 , 7}$ & $\mathbf{8 2 , 6}$ \\
Italia & 43,6 & 46,1 & $\mathbf{4 6 , 4}$ & $\mathbf{4 7 , 7}$ & $\mathbf{4 8 , 6}$ & 50,7 & 52,4 \\
Grecia & 15,0 & 16,4 & 15,5 & 17,9 & 22,0 & 23,6 & 24,8 \\
Croacia & 9,1 & 9,9 & 10,3 & 11,0 & 11,6 & 12,7 & 13,8 \\
Montenegro & 0,5 & 1,2 & 1,3 & 1,3 & 1,3 & 1,6 & 1,7 \\
Marruecos & 9,3 & 9,3 & 9,4 & 10,0 & 10,3 & 10,2 & 10,3 \\
Túnez & 6,9 & 4,8 & 5,9 & 6,3 & 6,1 & 5,4 & 5,7 \\
Egipto & 14,1 & 9,5 & 11,2 & 9,2 & 9,6 & 9,1 & 5,3 \\
Turquía & 27,0 & 29,3 & 35,7 & 37,8 & 39,8 & 39,5 & 25,3 \\
\hline
\end{tabular}

Fuente: UNWTO, UNWTO Tourism Highlights, 2011-2017. 
Es de destacar que la industria del turismo es un sector estratégico para la mayoría de los países mediterráneos, y especialmente para Túnez, Egipto, Marruecos y Turquía. Es una «columna vertebral» de sus economías (Perles-Ribes et al., 2018). Según el Foro Económico Mundial (2011-2017), esta industria representaba del 5 al 10\% del PIB. Al mismo tiempo, este sector proporcionó del 3 al 10\% del total de empleos. Con la crisis, el desempeño económico de la industria de viajes y turismo se redujo, particularmente en el norte de África y las partes del Medio Oriente del Mediterráneo. Los ingresos del turismo disminuyeron drásticamente. El mayor descenso en los ingresos se registró en Egipto (> 400\%), Túnez (> 200\%) y Turquía (160\%). En estas regiones, el turismo mostró una gran dependencia de los turistas europeos, que representaron más del $60 \%$ de la demanda total. De esta manera, una vez más, se destacaron los efectos de la propagación de la inestabilidad política en los países vecinos (Steiner, 2007).

\section{LA RELACIÓN ENTRE LOS PROBLEMAS DE SEGURIDAD Y LAS OPCIONES DE DESTINO}

En la industria del turismo, la seguridad y la protección a menudo se han usado indistintamente, incluso en otras disciplinas. Existe una clara distinción entre estos dos términos. La explicación podría encontrarse en la alta sensibilidad del turismo a cualquier tipo de interrupción (Tarlow, 2014). Durante el proceso de elección de un destino turístico, los turistas potenciales evalúan las alternativas de destino comparando los costos y beneficios del destino. Sönmez y Graefe (1998) propusieron un marco internacional para la toma de decisiones sobre viajes. Clasificaron los factores que influyen en la elección del destino en tres categorías: factores externos (medios de comunicación, consultorías de viajes gubernamentales e interaccio- nes sociales), factores internos (experiencias de viaje, opiniones y percepciones de seguridad) y factores demográficos. Además, Roehl y Fesenmaier (1992), así como Sönmez y Graeffe (1998), evaluaron nueve tipos de riesgos asociados con los viajes: riesgos financieros, de salud, físicos, sociales, riesgos psicológicos y temporales, satisfacción, inestabilidad política y terrorismo.

En las últimas décadas, el terrorismo ha estado fuertemente asociado con la industria del turismo. Desde 2001, con los ataques terroristas en los Estados Unidos, todo el concepto de turismo ha cambiado. Los funcionarios de viajes y turismo se han involucrado en asuntos de seguridad nacional e internacional. Como argumenta Tarlow (2014: 98), «el turismo ha sido un imán para los ataques terroristas". Hay varias razones por las cuales el turismo y los visitantes son atractivos para los terroristas. El turismo brinda oportunidades para ser víctimas masivas en lugares emblemáticos (Tarlow, 2014). Los turistas son vulnerables y fáciles de atacar. Son los llamados objetivos «blancos» porque durante las vacaciones, tienden a relajarse y son menos cautelosos con su entorno (Tarlow, 2006). En muchos casos, los objetivos son los turistas «occidentales». A «Occidente» generalmente se le percibe como un apoyador de los gobiernos débiles de los países islámicos y que intenta detener a los grupos radicales que luchan por ganar el dominio. Además, como los medios de comunicación son propiedad en su mayoría de corporaciones occidentales, «matar a ciudadanos occidentales tiene muchas más probabilidades de llamar la atención de los medios de comunicación globales» (Neumayer y Plümper, 2016). Además, su estilo de vida y comportamiento (el tipo de vestimenta, consumo de alcohol, juegos de azar, etc.) han sido condenados por terroristas. Hoy en día, un pequeño número de lugares puede considerarse seguro, lo que demuestra que los terroristas ya han logrado crear un clima de miedo en todo el mundo (Sönmez y Graefe, 1998). 
Los estudios empíricos realizados hasta ahora han demostrado que los problemas de seguridad y su impacto en las tendencias de demanda turística dependen de varios factores. En primer lugar, están los factores sociodemográficos, tales como el género (Pizam et al., 2004), la edad (Marion, 2018; Reisinger y Mavondo, 2006; Sönmez y Graefe, 1998) y la educación (Marion, 2018; Park y Reisinger, 2010; Sönmez y Graefe, 1998). Además, el sentimiento del riesgo también se ve afectado por viajar con niños pequeños (Sönmez y Graefe, 1998), por antecedentes culturales (Lepp y Gibson, 2003), por el tipo de personalidad (individuos con aversión al riesgo y buscadores de riesgo), y por la experiencia previa y la nacionalidad (especialmente entre visitantes occidentales) (Fuchs y Reichel, 2011). Sin embargo, hay hallazgos contradictorios teniendo en cuenta algunos factores. Por ejemplo, con respecto a la importancia de las diferencias relacionadas con el género en las percepciones del riesgo de viaje, algunos autores encontraron que el género no influye en la percepción del riesgo (Carr, 2001; Sönmez y Graefe, 1998), mientras que otros estudios señalaron el papel esencial de este factor (Elsrud, 2001; Enloe, 2014; Gustafson, 1998; Hawes, 1988; Wearing y Wearing, 1996). Muchos académicos coinciden en que la seguridad y la protección son factores esenciales que determinan la demanda turística y una condición previa para el éxito del turismo internacional (Ghaderi, Saboori y Khoshkam, 2017; Fletcher y Morakabati, 2008; Mansfeld y Pizam, 2006). Si los posibles riesgos de convertirse en víctima son mayores que los beneficios, es probable que los turistas cancelen sus viajes a pesar de la lealtad del destino (Altindag, 2014; Law, 2006). Basándose en el estado actual y los resultados de los estudios recientes, el objetivo principal y los objetivos especiales se definieron para aclarar el impacto de ciertos factores en la seguridad individual y la demanda turística internacional. El objetivo general de este estudio es determinar si la crisis política y de seguridad inducida por los acontecimientos en la región MENA afectó el comportamiento de los turistas serbios jóvenes y de mediana edad y en qué medida. Siguiendo esta pregunta general, cinco hipótesis específicas se han definido:

1. La percepción de seguridad depende del género.

2. Las preocupaciones sobre seguridad dependen de la edad y los ingresos.

3. La imagen mediática de la situación de seguridad actual aumenta la sensación de inseguridad de los turistas potenciales.

4. El comportamiento de viaje de la población de Serbia que es joven y de mediana edad ha cambiado durante la crisis.

5. La impresión de seguridad y protección en el destino es un factor decisivo en la toma de decisiones sobre dónde viajar.

\section{MÉTOdos Y RecopiLACIÓN DE dATOS}

El Mediterráneo ha sido el destino turístico mundial más importante debido a la mayor cantidad de llegadas de turistas y pernoctaciones. Además, durante los últimos años, se ha enfrentado a numerosos problemas de seguridad causados por la crisis de la Primavera Árabe. El estallido de las migraciones y la creación de la ruta importante de migración de los Balcanes occidentales que pasa por Serbia fue la razón principal por la que esta área fue elegida para nuestro estudio.

Adicionalmente, los países mediterráneos han sido tradicionalmente la principal opción de vacaciones para los turistas serbios, por lo que queríamos determinar si los problemas de seguridad inducidos políticamente influyeron en los cambios en el comportamiento de los turistas y en qué medida.

Este artículo se ha centrado en un segmento específico de la población, en los turistas jóvenes y de mediana edad (grupo de edad: $19-39$ ), que representan el $26,6 \%$ de la población serbia $(13,5 \%$ hombres, $13,1 \%$ mujeres). Varios estudios confirmaron que 
las preocupaciones de seguridad crecen con la edad (Gibson y Yiannakis, 2002; Sönmez y Graefe, 1998), por lo que queríamos examinar cómo este grupo percibe los riesgos y responde a las cuestiones de seguridad y protección, y para determinar si se aplican algunos principios diferentes y específicos a este sector.

El estudio se realizó utilizando como técnica de investigación una encuesta autoadministrada abierta en línea. Esta forma de recopilar datos en estudios de turismo ha demostrado ser adecuada, porque facilita la obtención de respuestas más completas y de calidad (Ballard y Prine, 2002; Dolnicar, Laesser y Matus, 2009). Es particularmente evidente cuando se trata de este grupo de edad específico de encuestados lo complicado que es llegar a comunicarse por otros métodos (por ejemplo, entrevistas personales, encuestas telefónicas, etc.) (Niemi, Portney y King, 2008).

Los datos fueron recopilados entre los residentes serbios durante junio y julio de 2016. Se recolectó la muestra de 301 encuestados, mientras que 249 respuestas se consideraron válidas para el estudio. Parte de los encuestados fueron excluidos de un análisis posterior porque no cumplían con el requisito del grupo de edad, pero también debido a algunas razones técnicas (respuestas incompletas y contradictorias, falta de datos, etc.) (tabla 2).

TABLA 2. Ficha técnica

\begin{tabular}{lc}
\hline Cuestionarios recogidos & 301 \\
Cuestionarios válidos & 249 \\
Tasa de respuesta & $82,72 \%$ \\
Nivel de confianza & $95 \%$ \\
Tipo de encuesta & auto-administrada \\
Encuesta completada & julio 2016 \\
Procedimientos estadísticos & SPSS estadísticas \\
\hline
\end{tabular}

Fuente: Elaboración propia.
El cuestionario final contaba con tres partes. La primera parte se centró en las características socioeconómicas y demográficas de los encuestados, alineado con la metodología oficial del censo de Serbia (2011). La segunda parte examinó los hábitos de viaje actuales de los ciudadanos serbios. En la tercera parte, se examinaron las actitudes de los turistas que pasaron sus vacaciones en los destinos mediterráneos afectados por numerosos riesgos de seguridad. El cuestionario incluyó varios tipos de cuestionamientos: preguntas abiertas, preguntas cerradas y de escala Likert. Los valores en la escala Likert variaron de 1 (totalmente en desacuerdo) a 5 (totalmente de acuerdo). El Paquete Estadístico para las Ciencias Sociales (versión 21.0) fue utilizado para el procesamiento de datos. Finalmente, en el análisis de datos, se utilizaron dos pruebas estadísticas para la comparación de grupos (prueba de ji-cuadrado y la prueba de la $U$ de Mann-Whitney). Teniendo en cuenta que se violaron los requisitos previos para la correlación de Pearson (normalidad, homocedasticidad y linealidad), aplicamos un análisis de correlación no paramétrico.

\section{Resultados Y discusión}

\section{Descripción de la muestra}

Como se discutió anteriormente, esta investigación se refiere a 249 turistas serbios jóvenes y de mediana edad $(82,7 \%$ de respuestas válidas). La mayoría de los encuestados eran mujeres $(66,7 \%)$. En cuanto a la estructura de edad, los encuestados del grupo de 19 a 29 representaron el 69,1\%, mientras que el grupo de 30 a 39 años representó el 30,9\%. Esto indica que la población «más joven» estaba más dispuesta a participar en la investigación. Casi el $80 \%$ de los encuestados tenía educación superior y el $53 \%$ estaba empleado. Más del 
$20 \%$ no tenía ingresos y casi el $30 \%$ tenía ganancias que iban desde 25.000 RSD (alrededor de 200 EUR) hasta 50.000 RSD (alrededor de 450 EUR). Casi el $15 \%$ de los encuestados no quería responder a la pregunta sobre los ingresos mensuales porque lo consideraban extremadamente personal (tabla 3).

TABLA 3. Características sociodemográficas de los encuestados

\begin{tabular}{|c|c|c|}
\hline Demografía & Frecuencias & $\begin{array}{l}\text { Porcentaje } \\
\text { (\%) }\end{array}$ \\
\hline \multicolumn{3}{|l|}{ Género } \\
\hline Mujeres & 166 & 66,7 \\
\hline Hombres & 83 & 33,3 \\
\hline \multicolumn{3}{|l|}{ Edad } \\
\hline $19-29$ & 172 & 69,1 \\
\hline $30-39$ & 77 & 30,9 \\
\hline \multicolumn{3}{|l|}{ Nivel de educación } \\
\hline Escuela secundaria & 53 & 21,3 \\
\hline Titulo académico & 196 & 78,7 \\
\hline \multicolumn{3}{|l|}{ Ocupación } \\
\hline Estudiante & 91 & 36,5 \\
\hline Empleado & 132 & 53,0 \\
\hline Desempleado & 26 & 10,5 \\
\hline \multicolumn{3}{|l|}{$\begin{array}{l}\text { Ingresos mensuales } \\
\text { (en euros) }\end{array}$} \\
\hline Sin ingresos & 55 & 22,1 \\
\hline$<200$ & 28 & 11,2 \\
\hline $201-450$ & 68 & 27,3 \\
\hline $451-650$ & 31 & 12,4 \\
\hline$>650$ & 33 & 13,3 \\
\hline $\begin{array}{l}\text { No quiero responder } \\
\text { esta pregunta }\end{array}$ & 34 & 13,7 \\
\hline TOTAL & 249 & 100,0 \\
\hline
\end{tabular}

Fuente: Elaboración propia.

\section{Los desafíos políticos y las actitudes de los turistas}

Se pidió a los encuestados que expresaran sus actitudes hacia las declaraciones relacionadas con los problemas actuales de seguridad y su impacto en el riesgo percibido en los destinos turísticos.

Demostraron un alto nivel de conocimiento de los fenómenos clave en este campo. Casi el $80 \%$ estaba familiarizado con los términos "Estado Islámico» y «Ruta de Migración de los Balcanes», mientras que solo el 2,8\% no tenía suficiente información. En la tabla 4 se presentan las actitudes de los turistas sobre ciertos desafíos. En cuanto a la declaración «Las operaciones de ISIS perturban la industria del turismo de los países mediterráneos", la mayoría de los encuestados o el $72,2 \%$ expresó su acuerdo. Además, hubo una alta tasa de respuestas neutrales $(18,5 \%)$, mientras que una minoría no estuvo de acuerdo (totalmente en desacuerdo $2,4 \%$, y en desacuerdo $6,8 \%)$. Las respuestas a la segunda declaración «La ruta de migración de los Balcanes afecta el proceso de toma de decisiones" fueron bastante equilibradas: no está de acuerdo un $31,3 \%$, es neutral el $34,9 \%$ y el $33,7 \%$ sí está de acuerdo. Con respecto a la declaración, "La alta densidad de población migrante representa el riesgo de enfermedades contagiosas", más del $44 \%$ estuvo de acuerdo con la verificación, lo que hace que las neutrales sean casi el $80 \%$. La cuarta declaración «La nueva política de gestión de fronteras influye negativamente en la elección del destino", mostró que los turistas potenciales que estuvieron de acuerdo con la declaración representaron el $48 \%$. Los encuestados expresaron una actitud predominantemente neutral $(53 \%)$ hacia la declaración «Los servicios de seguridad de los países mencionados invierten los máximos esfuerzos para mejorar 
la seguridad en los destinos». Finalmente, casi el $80 \%$ de los encuestados estuvo de acuerdo en que «La imagen de los medios de comunicación sobre la situación de seguridad actual aumenta la sensación de inseguridad en los turistas potenciales».

TABLA 4. Frecuencias de actitudes turísticas (\%)

\begin{tabular}{|c|c|c|c|c|c|}
\hline Declaraciones & 1 & 2 & 3 & 4 & 5 \\
\hline $\begin{array}{l}\text { Las operaciones de ISIS perturban la industria del turismo de los paí- } \\
\text { ses mediterráneos. }\end{array}$ & 2,4 & 6,8 & 18,5 & 36,1 & 36,1 \\
\hline $\begin{array}{l}\text { La ruta de migración de los Balcanes afecta el proceso de toma de de- } \\
\text { cisiones. }\end{array}$ & 10,8 & 20,5 & 34,9 & 21,3 & 12,4 \\
\hline $\begin{array}{l}\text { La alta densidad de población migrante representa el riesgo de enfer- } \\
\text { medades contagiosas. }\end{array}$ & 6,8 & 14,9 & 34,1 & 22,9 & 21,3 \\
\hline $\begin{array}{l}\text { La nueva política de gestión de fronteras influye negativamente en la } \\
\text { elección del destino. }\end{array}$ & 11,2 & 17,7 & 23,3 & 29,7 & 18,1 \\
\hline $\begin{array}{l}\text { Los servicios de seguridad de los países mencionados invierten los } \\
\text { máximos esfuerzos para mejorar la seguridad en los destinos. }\end{array}$ & 6,0 & 13,3 & 53,0 & 21,7 & 6,0 \\
\hline $\begin{array}{l}\text { La imagen de los medios de comunicación sobre la situación de segu- } \\
\text { ridad actual aumenta la sensación de inseguridad en los turistas po- } \\
\text { tenciales. }\end{array}$ & 1,6 & 4,8 & 16,5 & 43,4 & 33,7 \\
\hline
\end{tabular}

Nota: 1 = totalmente en desacuerdo, 2 = en desacuerdo, 3 = neutral, 4 = de acuerdo, 5 = totalmente de acuerdo.

Fuente: Elaboración propia.

Para identificar diferencias en las actitudes hacia las declaraciones mencionadas anteriormente entre hombres/mujeres y entre diferentes grupos de edad, se aplicó una prueba de ji-cuadrado para la independencia (tabla 5). Teniendo en cuenta que el estudio incluyó variables con más de dos categorías, el coeficiente $\checkmark$ de Cramer se utilizó para medir la fuerza de la relación entre las variables categóricas. La prueba de ji-cuadrado reveló una relación importante entre la distribución de las actitudes de los encuestados y su género con respecto a las declaraciones "Impacto de las operaciones de ISIS en la industria del turismo" $X^{2}(4, n=247)=$ $12,91, p=0,01$, phi $=0,23$; «La alta densidad de población migrante representa el riesgo de enfermedades contagiosas" $X^{2}$ $(4, \mathrm{n}=247)=10,50, p=0,03$, phi $=0,20$; y «La nueva política de gestión de fronteras influye negativamente en la elección del destino" $X^{2}(4, n=249)=13,21$, $p=0,01$, phi $=0,22$, con una probabilidad del $5 \%(p \leq 0,05)$. En casos observados, el coeficiente de Cramer (V) muestra una fuerza de correlación moderada (aceptable) entre las variables. En el caso de «La imagen de los medios de comunicación sobre la situación de seguridad actual aumenta la sensación de inseguridad en los turistas potenciales", el ji-cuadrado fue $X^{2}$ $(4, n=267)=9,28, p=0,05$, phi $=0,19$, pero el coeficiente de Cramer sugiere una fuerza de correlación débil entre las variables. Las mujeres proporcionaron respuestas (acuerdos) más neutrales y positivas que los hombres. Entre todas las variables enumeradas, se encontró una correlación entre la edad de los encues- 
tados y la afirmación «Los servicios de seguridad de los países mencionados invierten los máximos esfuerzos para mejorar la seguridad en los destinos» $X^{2}$ (4, $\mathrm{n}=267)=7,22, p=0,05$. El valor de Cramer $(0,14)$ indica una correlación débil. En este caso, el porcentaje entre quienes no estaban de acuerdo con la declaración fue mayor $(19,3 \%)$ en el grupo más joven (19-29). Curiosamente, el grupo 30-39 se abstuvo más en responder a preguntas (53\% de respuestas neutrales).

TABLA 5. Los resultados de la prueba de ji-cuadrado para la independencia

\begin{tabular}{|c|c|c|c|}
\hline Declaraciones & & $\begin{array}{l}\text { Chi-square } \\
\text { (p valor) }\end{array}$ & $\begin{array}{l}\text { Coeficiente V } \\
\text { de Cramer }\end{array}$ \\
\hline $\begin{array}{l}\text { Las operaciones de ISIS perturban la industria del turismo de } \\
\text { los países mediterráneos. }\end{array}$ & $\begin{array}{l}\text { género } \\
\text { edad }\end{array}$ & $\begin{array}{l}0,012^{\star \star} \\
0,379\end{array}$ & $\begin{array}{l}0,229 \\
0,130\end{array}$ \\
\hline $\begin{array}{l}\text { La ruta de migración de los Balcanes afecta el proceso de toma } \\
\text { de decisiones. }\end{array}$ & $\begin{array}{l}\text { género } \\
\text { edad }\end{array}$ & $\begin{array}{l}0,095 \\
0,372\end{array}$ & $\begin{array}{l}0,178 \\
0,131\end{array}$ \\
\hline $\begin{array}{l}\text { La alta densidad de población migrante representa el riesgo de } \\
\text { enfermedades contagiosas. }\end{array}$ & $\begin{array}{l}\text { género } \\
\text { edad }\end{array}$ & $\begin{array}{l}0,033^{* *} \\
0,227\end{array}$ & $\begin{array}{l}0,205 \\
0,151\end{array}$ \\
\hline $\begin{array}{l}\text { La nueva política de gestión de fronteras influye negativamente } \\
\text { en la elección del destino. }\end{array}$ & $\begin{array}{l}\text { género } \\
\text { edad }\end{array}$ & $\begin{array}{l}0,014^{*} \\
0,158\end{array}$ & $\begin{array}{l}0,224 \\
0,163\end{array}$ \\
\hline $\begin{array}{l}\text { Los servicios de seguridad de los países mencionados invierten } \\
\text { los máximos esfuerzos para mejorar la seguridad en los desti- } \\
\text { nos. }\end{array}$ & $\begin{array}{l}\text { género } \\
\text { edad }\end{array}$ & $\begin{array}{l}0,696 \\
0,055^{\star \star}\end{array}$ & $\begin{array}{l}0,094 \\
0,170\end{array}$ \\
\hline $\begin{array}{l}\text { La imagen de los medios de comunicación sobre la situación } \\
\text { de seguridad actual aumenta la sensación de inseguridad en los } \\
\text { turistas potenciales. }\end{array}$ & $\begin{array}{l}\text { género } \\
\text { edad }\end{array}$ & $\begin{array}{l}0,054^{* *} \\
0,690\end{array}$ & $\begin{array}{l}0,193 \\
0,095\end{array}$ \\
\hline
\end{tabular}

Nota: ${ }^{*} p \leq 0,01 ;{ }^{* \star} p \leq 0,05$.

Fuente: Elaboración propia.

Los autores también compararon las medianas entre dos grupos independientes (hombres y mujeres) usando una prueba de la U de Mann-Whitney (tabla 6). Como una versión no paramétrica de la prueba «t» para muestras independientes, se evalúa si los rangos de los dos grupos difieren significativamente. Los resultados revelaron que había una diferencia significativa entre los géneros con respecto a las actitudes hacia las declaraciones «La ruta de migración de los Balcanes afecta el proceso de toma de decisiones» y «La imagen de los medios de comunicación sobre la situación de seguridad actual au- menta la sensación de inseguridad en los turistas potenciales». En la primera afirmación $U=5695, z=-2,30, p=0,002$, con la misma mediana para hombres y mujeres $(M d m f=3, n m=83, n f=166)$. El significado para la segunda afirmación fue $0,01(U=5553, z=-2,66, p=0,008)$ con (Md $=4, n=83$ ) para los hombres y $(M d=4, n=166)$ para las mujeres. Adicionalmente, calculamos el valor estadístico del tamaño del efecto $(r)$ en función del valor $z$ y el número total de casos. El valor $r$ indica un tamaño pequeño del efecto $(0,15$ para la primera y 0,17 para la segunda afirmación). 
TABLA 6. Los resultados de la prueba de la U de Mann-Whitney

\begin{tabular}{lccc}
\hline \multicolumn{1}{c}{ Declaraciones } & U prueba & $\boldsymbol{z}$ & $\boldsymbol{p}$ \\
\hline $\begin{array}{l}\text { Las operaciones de ISIS perturban la industria del turismo de los paí- } \\
\text { ses mediterráneos. }\end{array}$ & 6.385 & $-0,992$ & 0,321 \\
$\begin{array}{l}\text { La ruta de migración de los Balcanes afecta el proceso de toma de } \\
\text { decisiones. }\end{array}$ & 5.695 & $-2,304$ & $0,021^{\star \star}$ \\
$\begin{array}{l}\text { La alta densidad de población migrante representa el riesgo de en- } \\
\text { fermedades contagiosas. }\end{array}$ & 5.921 & $-1,869$ & 0,062 \\
$\begin{array}{l}\text { La nueva política de gestión de fronteras influye negativamente en la } \\
\text { elección del destino. }\end{array}$ & 5.894 & $-1,907$ & 0,057 \\
$\begin{array}{l}\text { Los servicios de seguridad de los países mencionados invierten los } \\
\text { máximos esfuerzos para mejorar la seguridad en los destinos. }\end{array}$ & 6.325 & $-1,150$ & 0,250 \\
$\begin{array}{l}\text { La imagen de los medios de comunicación sobre la situación de se- } \\
\text { guridad actual aumenta la sensación de inseguridad en los turistas } \\
\text { potenciales. }\end{array}$ & 5.553 & $-2,665$ & $0,008^{\star}$ \\
\hline
\end{tabular}

Nota: ${ }^{*} p \leq 0,01 ;{ }^{* *} p \leq 0,05$.

Fuente: Elaboración propia.

TABLA 7. Los resultados del análisis de correlación

\begin{tabular}{|c|c|c|c|c|}
\hline Declaraciones & & Género & Edad & $\begin{array}{l}\text { Ingresos } \\
\text { mensuales }\end{array}$ \\
\hline \multirow{2}{*}{$\begin{array}{l}\text { Las operaciones de ISIS perturban la industria del turismo de los } \\
\text { países mediterráneos. }\end{array}$} & rho & $-0,063$ & 0,048 & 0,021 \\
\hline & $p$ & 0,322 & 0,048 & 0,742 \\
\hline \multirow{2}{*}{$\begin{array}{l}\text { La ruta de migración de los Balcanes afecta el proceso de toma } \\
\text { de decisiones. }\end{array}$} & rho & $-0,146$ & 0,019 & $-0,009$ \\
\hline & $p$ & $0,021^{* *}$ & 0,769 & 0,891 \\
\hline \multirow{2}{*}{$\begin{array}{l}\text { La alta densidad de población migrante representa el riesgo de } \\
\text { enfermedades contagiosas. }\end{array}$} & rho & $-0,119$ & $-0,115$ & $-0,093$ \\
\hline & $p$ & 0,062 & 0,069 & 0,144 \\
\hline \multirow{2}{*}{$\begin{array}{l}\text { La nueva política de gestión de fronteras influye negativamente } \\
\text { en la elección del destino. }\end{array}$} & rho & $-0,121$ & $-0,137$ & $-0,035$ \\
\hline & $p$ & 0,056 & $0,030^{* *}$ & 0,588 \\
\hline \multirow{2}{*}{$\begin{array}{l}\text { Los servicios de seguridad de los países mencionados invierten } \\
\text { los máximos esfuerzos para mejorar la seguridad. en los destinos. }\end{array}$} & rho & $-0,073$ & 0,082 & 0,016 \\
\hline & $p$ & 0,251 & 0,198 & 0,799 \\
\hline \multirow{2}{*}{$\begin{array}{l}\text { La imagen de los medios de comunicación sobre la situación de } \\
\text { seguridad actual aumenta la sensación de inseguridad en los tu- } \\
\text { ristas potenciales. }\end{array}$} & rho & $-0,169$ & $-0,020$ & $-0,122$ \\
\hline & $p$ & $0,007^{\star}$ & 0,757 & $0,050^{* *}$ \\
\hline
\end{tabular}

Nota: rho de Spearman; ${ }^{*} p \leq 0,01 ;{ }^{* *} p \leq 0,05$.

Fuente: Elaboración propia.

Reis. Rev.Esp.Investig.Sociol. ISSN-L: 0210-5233. N. ${ }^{\mathbf{1}}$ 174, Abril - Junio 2021, pp. 69-88 
En la secuela, aplicamos el análisis de correlación no paramétrico de Spearman. Se examinó la relación entre las variables independientes (género, edad e ingresos mensuales) y las respuestas obtenidas de la escala Likert de cinco puntos como variable dependiente (tabla 7). Los resultados revelaron la correlación estadísticamente significativa entre varias declaraciones: «La ruta de migración de los Balcanes afecta el proceso de toma de decisiones» y el género; «La nueva política de gestión de fronteras influye negativamente en la elección del destino" y la edad; y «La imagen de los medios de comunicación sobre la situación de seguridad actual aumenta la sensación de inseguridad en los turistas potenciales» y el género y los ingresos mensuales. En todos los casos analizados, se registró una pequeña correlación negativa.

\section{El impacto de la crisis política en el comportamiento de los viajes}

Además, para determinar si los hábitos de viaje de la población joven y de mediana edad de Serbia cambiaron, también analizamos el estado antes y durante la crisis. La mayoría de los encuestados (más del 70\%) confirmaron que viajaron durante el período 2013-2015. Entre ellos, dos tercios los for- maban mujeres entre 19 y 29 años, con un alto nivel de educación y empleo, con un ingreso promedio de 200 a 450 euros. Los resultados mostraron que la mayoría de los serbios jóvenes y de mediana edad visitaron los siguientes destinos: Grecia (37,7\%), Montenegro (25,7\%), Turquía $(9,9 \%)$ y Croacia $(8,0 \%)$. Una parte más pequeña de los encuestados pasaron sus vacaciones en Italia $(3,8 \%)$, Egipto $(3,3 \%)$, España $(3,3 \%)$ y Túnez (0,7\%). Los resultados de nuestra encuesta en su mayoría coinciden con los datos oficiales que se muestran en la tabla 8 (Statistical Office of the Republic of Serbia, 2011-2016). Los datos muestran el número de turistas que utilizaron paquetes organizados por agencias de viajes locales desglosados por destino, excepto para Croacia. La Oficina de Estadística no proporciona datos sobre el porcentaje de turistas que viajaron a Croacia a través de agencias de viajes porque un pequeño número de agencias de viajes serbias ofrecen paquetes de vacaciones para Croacia y la mayoría de los turistas organizan sus viajes individualmente, como lo confirmaron nuevamente los resultados de nuestra encuesta. Adicionalmente y por la misma razón, el número total de turistas serbios que viajan a Grecia y Montenegro se presenta en la tabla 9.

TABLA 8. Número de llegadas de turistas procedentes de Serbia en 2010-2015: los destinos principales de viaje

\begin{tabular}{lcccccc}
\hline Destino & $\mathbf{2 0 1 0}$ & $\mathbf{2 0 1 1}$ & $\mathbf{2 0 1 2}$ & $\mathbf{2 0 1 3}$ & $\mathbf{2 0 1 4}$ & $\mathbf{2 0 1 5}$ \\
\hline Grecia & 213.488 & 259.283 & 261.467 & 359.894 & 368.483 & 400.575 \\
Montenegro & 66.429 & 70.180 & 73.999 & 75.117 & 65.453 & 60.992 \\
Croacia & - & - & - & - & - & - \\
Turquía & 51.565 & 56.167 & 65.863 & 66.589 & 66.692 & 62.089 \\
Egipto & 32.483 & 22.455 & 25.264 & 11.656 & 18.083 & 6.102 \\
Túnez & 25.808 & 11.808 & 18.558 & 14.656 & 11.683 & 8.157 \\
España & 17.746 & 26.372 & 26.533 & 26.692 & 26.232 & 23.432 \\
Italia & 27.713 & 28.488 & 27.246 & 25.082 & 29.469 & 39.988 \\
\hline
\end{tabular}

Fuente: Oficina de Estadística de la República de Serbia, 2011-2016. 
TABLA 9. Número total de llegadas de turistas procedentes de Serbia 2010-2015

\begin{tabular}{lrrrrrc}
\hline Destino & \multicolumn{1}{c}{$\mathbf{2 0 1 0}$} & \multicolumn{1}{c}{$\mathbf{2 0 1 1}$} & $\mathbf{2 0 1 2}$ & $\mathbf{2 0 1 3}$ & \multicolumn{1}{c}{$\mathbf{2 0 1 4}$} & \multicolumn{1}{c}{$\mathbf{2 0 1 5}$} \\
\hline Grecia & 1.097 .505 & 1.099 .377 & 620.450 & 778.765 & 985.661 & 727.831 \\
Montenegro & 314.836 & 301.094 & 337.245 & 303.135 & 287.620 & 372.912 \\
Croacia & 87.000 & 87.000 & 86.000 & 89.768 & 94.085 & 101.397 \\
\hline
\end{tabular}

Fuente: Oficina de Estadística de Croacia, 2011-2016; Autoridad Estadística Helénica, 2011-2016; Oficina de Estadística de Montenegro, 2011-2016.

Como Altindag (2014) y Law (2006) argumentaron que el riesgo potencial podría llevar a la cancelación del viaje, se analizaron las intenciones de viaje para las vacaciones de verano en el año 2016. Aproximadamente el $63 \%$ respondió afirmativamente que viajarían. Entre los turistas que decidieron viajar, la mayoría eran mujeres de entre 19 y 29 años (más del 40\%). La mayoría de ellos eran altamente educados (> 50\%), empleados (35\%) y con un ingreso promedio de 200-450 euros. Cabe señalar que una gran parte de los encuestados no decidieron en el momento de la encuesta $(18,1 \%)$, mientras que el $18,9 \%$ no tenía intención de viajar durante el verano de 2016. En comparación con los años anteriores, se produjeron algunos cambios con respecto a la selección de destinos en 2016. Los resultados mostraron que los dos principales destinos seguían siendo Grecia (36,9\%) y Montenegro (7,6\%). Turquía había estado en el tercer lugar entre los turistas serbios durante el período anterior a la crisis, pero cayó al sexto lugar (1,2\%). Croacia (3,6\%), España e Italia $(2,8 \%)$ ascendieron un lugar. El cambio más significativo fue para los destinos del norte de África, Egipto y Túnez, que ya no estaban en la lista de los diez principales destinos entre los turistas serbios jóvenes y de mediana edad. En 2016, su participación era inferior al 0,5\%.

La República de Serbia formaba parte de la República Federativa Socialista de Yugoslavia, junto con Bosnia y Herzegovina, Croacia, Macedonia del Norte, Monte- negro y Eslovenia. El turismo fue un factor importante en las políticas sociales y nacionales, y el concepto de un Estado socialista. Mejorando los niveles de vida, este fenómeno social se convirtió en una característica importante del estilo de vida y de la identidad de los ciudadanos yugoslavos. Ofreció una nueva experiencia de recreación y vacaciones, con un tipo específico de consumismo a través de visitas organizadas, acciones laborales voluntarias juveniles y posesión masiva de propiedades (por ejemplo, casa de fin de semana, vikendica en serbio) (Grandits y Taylor, 2010). Después de la caída del régimen socialista, la República de Serbia continuó formando parte del Estado federal hasta 2006, junto con Montenegro. La cercanía cultural (el mismo idioma, religión e historia), la proximidad del destino y el acceso a la costa del Adriático son las razones principales por las cuales los turistas serbios todavía tienen la costumbre de pasar las vacaciones en este país. Hasta la década de los años noventa, los turistas serbios visitaban con frecuencia Croacia. En 1986, los turistas de Serbia realizaron más de seis millones de pernoctaciones en Croacia (Kobašić, 1987), frente a solo 477.000 en 2016 (Croatian Bureau of Statistics, 2017). Una de las razones fue la guerra civil yugoslava en los años noventa, que condujo a la ruptura de la RFSY y provocó tensiones entre los dos países. La mayor parte de los turistas se dirigieron hacia Grecia, y una parte mucho más pequeña hacia el vecino del sur de Serbia, Monte- 
negro. Más allá de estos temas políticos, hermosas costas, una cultura similar, buenos servicios y precios asequibles son las razones por las que Grecia se ha convertido en un destino preferido por los turistas serbios. Aunque las islas de Grecia oriental (por ejemplo, Samos, Lesbos) se vieron afectadas por una grave crisis migratoria desde 2015 y las reservas para estas islas se redujeron hasta un 90\% en 2016 (Angelopoulou y Roeder, 2016), Grecia se mantuvo como el destino número uno entre los jóvenes serbios y de mediana edad. A pesar de la crisis en las islas del Egeo oriental, los turistas serbios percibieron que, en las partes del norte, donde suelen tomar vacaciones, están a salvo.

Al mismo tiempo, Turquía se elevó como un destino relativamente nuevo entre los turistas serbios. Su parte como destino turístico estaba creciendo constantemente en el período 2008-2014. La inestabilidad política y los ataques terroristas llevaron a una disminución en el número de turistas serbios que visitan Turquía. El descenso más destacado se registró en 2015-2016 debido a dos ataques terroristas y a un intento de golpe de estado en Ankara y Estambul en 2016. Estos eventos profundizaron la crisis. Además, la posición geográfica de Turquía y la proximidad de Siria e Iraq aumentaron la ansiedad de los turistas potenciales, por lo que evitaban este destino (Teoman, 2017). Por otra parte, la demanda turística en los países del norte de África ha disminuido en los últimos años debido a la inestabilidad política y los ataques terroristas, de los cuales algunos estaban dirigidos a los turistas occidentales (por ejemplo, Egipto: 1997, 2004-2005, 2009, 2011 , 2015-2016; Túnez: 2002, 2013, 2015-2016). Durante estos años, muchos viajes combinados fueron cancelados, mientras que los principales lugares de interés fueron abandonados (Tomazos, 2017).

Por último, hemos tratado de determinar en qué medida la inestabilidad política y el terrorismo en los destinos influyeron en el proceso de toma de decisiones de los turistas. Solo el $30 \%$ de los encuestados confirmó que los acontecimientos recientes afectaron firmemente a sus decisiones de viaje. En su mayoría son las mujeres del grupo de edad de 19 a 29 años, empleadas (con ingresos de 200 a 450 euros) o estudiantes. Estos encuestados explicaron por qué los ataques terroristas en Turquía, Egipto y Túnez y toda la inestabilidad política en la región MENA afectaron su elección de destino. En el período anterior, las autoridades publicaron advertencias sobre la intención de viajar a estos países; los medios de comunicación informaron sobre los acontecimientos turbulentos en el área mencionada; siguiendo las recomendaciones de las autoridades, solo un número pequeño de agencias ofrecieron arreglos de viaje para los destinos mencionados. Incluso aquellos que no planearon viajar durante 2016, habrían evitado los países del norte de África y Turquía. La opinión más común entre los que tomaban vacaciones familiares era:

Aunque existen acuerdos muy favorables para Egipto, Túnez y Turquía, acciones terroristas (accidentes aéreos, ataques en balnearios y museos), así como una imagen mediática de la seguridad de los turistas nos disuadió de visitar estos países (ID 208).

Además, algunos consideraron lo siguiente:

Cuando se trata de elegir un destino, definitivamente no elegiría los países de la cultura islámica, porque creo que allí hay una mayor concentración de extremistas, por lo que probablemente me sentiría muy inseguro en cualquier momento y a cada paso. ¿Por qué viajaría a algún lugar con temor, donde existe la posibilidad de que algo me explote? (ID 216).

Desafortunadamente, esta opinión está en línea con la conclusión hecha en el trabajo de Desrues (2009: 9) que «los musulmanes en su conjunto son percibidos como islamistas». 
También, un alto porcentaje de los encuestados $(70 \%)$ enfatizó que la situación de seguridad no afectó a sus decisiones porque tradicionalmente viajaron a Grecia y Montenegro, los cuales ellos consideraron seguros. Algunas de las muchas opiniones similares fueron:

Creo que los griegos harán más con respeto a la seguridad porque un posible ataque terrorista destruiría la temporada y al mismo tiempo rompería el presupuesto que depende en gran medida del turismo (ID 221).

\section{$\mathrm{y}$}

El año pasado, durante la crisis migratoria, todo salió bien, así que no hay razón para evitarlo este año (ID 146).

Una parte de los encuestados creía que se deberían elegir los destinos menos conocidos, que no atraen a un gran número de turistas, ya que estarían menos expuestos al riesgo y al impacto del terrorismo:

En esencia, los destinos populares de los turistas occidentales deberían ser evitados. Esto incluye los lugares que eran o fueron anunciados a ser blanco de los terroristas (por ejemplo, Egipto, Túnez, Turquía, pero también Francia e Italia) (ID 62).

Al final, este estudio revela que hay un pequeño grupo así llamado «buscadores de riesgo». Por lo general, les preocupa menos la seguridad y protección en el destino durante la toma de decisiones (Sönmez y Graefe, 1998). Varios encuestados declararon que no estaban interesados en posibles ataques terroristas: "No me importan esas cosas en absoluto, los ataques terroristas no pueden asustarme» (ID 100) o «No creo que la situación sea tan crítica, además, usaría los arreglos baratos para Egipto y Túnez» (ID 244).

El estudio mostró que la seguridad es un factor importante, pero no decisivo, en el proceso de selección de destinos para los turistas serbios jóvenes y de mediana edad. Los factores más importantes son el precio para la mayoría de ellos, seguido de la calidad y la seguridad del servicio. Teniendo en cuenta el poder económico limitado de los habitantes serbios, el segmento especialmente investigado de la demanda turística, podría entenderse por qué el precio del viaje es el factor más significativo (Micić, Denda y Popescu, 2019).

\section{Conclusiones}

La reciente crisis de seguridad en la región mediterránea tuvo varias consecuencias a nivel local y global. Se reflejan en los cambios de régimen en los países de la región MENA, las migraciones, el fortalecimiento de los movimientos extremistas, los ataques terroristas y la propagación del clima de temor, que sin duda ha tenido un impacto en la industria del turismo. Altindag (2014) y Neumayer (2004) señalaron que la seguridad personal es un factor importante en la elección del destino, por lo que, si el riesgo percibido es mayor, los turistas potenciales están dispuestos a reconsiderar su elección de destino. Fourie, Roselló-Nadal y Santana-Gallego (2020) argumentaron que el terrorismo y la delincuencia tienen un impacto negativo en el turismo entrante.

Los resultados de nuestro estudio revelaron que los turistas jóvenes y de mediana edad de Serbia son conscientes de la crisis actual en la región MENA. En su mayoría están de acuerdo en que la Primavera Árabe y los acontecimientos relacionados influyeron negativamente en el desarrollo del turismo en la región y que los medios de comunicación empeoran su sensación de seguridad. Una vez más, se confirma que los medios tienen un papel importante durante el período de crisis porque transmiten la información sobre la situación de seguridad. El problema que enfrentan los destinos turísticos es la exageración que puede dañar la imagen del destino que podría durar años (Ghaderi, Saboori y Khoshkam, 2017; Tomazos, 2017). Los medios 
de comunicación ven los ataques terroristas, la inestabilidad política, las guerras, etc., como una fuente de noticias valiosa que podría elaborarse con detalles con credibilidad cuestionable (Ghaderi, Saboori y Khoshkam, 2017; Mansfeld y Pizam, 2006; Teoman, 2017).

Muchos estudios han demostrado que la percepción del riesgo depende del género, la edad, el nivel educativo, el tipo de personalidad, la nacionalidad y la experiencia previa en viajes (Fuchs y Reichel, 2011; Lepp y Gibson, 2003; Marion, 2018; Pizam et al., 2004; Sönmez y Graefe, 1998). A pesar de los hallazgos de Carr (2001), Lepp y Gibson (2003) y Sönmez y Graefe (1998), se determinó la diferencia significativa entre géneros, por lo que este tiene un papel importante en la percepción del riesgo. Aparte de eso, una correlación débil entre grupos de edad e ingresos relacionados con problemas de seguridad.

Además, los resultados de la investigación realizada entre los turistas serbios jóvenes y de mediana edad confirmaron que se produjeron algunos cambios en el comportamiento de los viajes y la reubicación de la demanda turística durante el estallido de la crisis migratoria causada por la Primavera Árabe. La agitación política y los ataques terroristas en Turquía y los destinos del norte de África (Egipto y Túnez) dañaron la imagen del destino y aumentaron el riesgo percibido entre los turistas, por lo que estos destinos fueron evitados por una parte de la población serbia más joven. Es evidente que el terrorismo disminuye el turismo entrante y daña la industria del turismo (Drakos y Kutan, 2003; Llorca-Vivero, 2008).

Aunque varios estudios (Morakabati, 2007; Reisinger y Mavondo, 2005) confirmaron que la seguridad y la protección son principales factores de motivación en el proceso de decisión de viaje, los resultados de nuestro estudio mostraron que solo la parte más pequeña de los encuestados tiende a evitar los destinos que se enfrentan a problemas de seguridad. Por lo tanto, la mayoría de los encuestados consideraron que la seguridad es de gran importancia, pero no decisiva en la selección del destino y en tomar la decisión de viajar o no. Esta actitud podría explicarse por la inestabilidad política y las guerras a las que se enfrentaron los habitantes serbios. La inestabilidad en su país de origen puede ser un factor en su mayor tolerancia hacia la inestabilidad política, la delincuencia y el terrorismo en posibles países de destino (Fourie, Roselló-Nadal y Santana-Gallego, 2020).

La otra razón es ciertamente el bajo poder económico de los ciudadanos serbios. Entre aquellos que no planearon viajar durante el verano de 2016, destacaron que ciertamente no habrían elegido destinos marcados como inseguros, como Turquía, Egipto y Túnez. Esta opinión está especialmente extendida entre las familias con bebés y niños pequeños. Una estrategia para superar este problema es viajar al destino turístico menos popular, lo que implica la asignación de la demanda turística.

Dado que la encuesta se realizó entre el segmento específico de los habitantes serbios y se refiere a una crisis particular, los resultados podrían generalizarse a poblaciones similares. En cualquier caso, los resultados de este estudio facilitan la comprensión del papel de la seguridad y protección en la toma de decisiones de viaje y la selección del destino, específicamente entre la población joven y de mediana edad. Estos hallazgos también podrían ayudar a los legisladores a comprender la importancia de la seguridad y protección para la imagen de destino. Es necesario enfatizar que las partes interesadas en todos los niveles deben trabajar juntas para reducir la inseguridad en el destino, debido a su vulnerabilidad a los problemas de seguridad (Fourie, RoseIló-Nadal y Santana-Gallego, 2020). 
En circunstancias contemporáneas, este tipo de investigación puede llevarse a cabo en diversos territorios que enfrentan problemas similares y como punto de partida para un análisis empírico adicional tanto en turismo como en estudios de seguridad. La investigación futura debe cubrir otras características socioeconómicas, étnicas, religiosas y personales de los encuestados y su actitud frente a los factores de riesgo. Como el estudio realizado por Ghaderi, Saboori y Khoshkam (2017) sugirió que la seguridad física es solo un factor de seguridad del destino turístico, otros también deberían ser investigados, como los derechos humanos, la bioseguridad y la seguridad ambiental. Al final, el nuevo estudio podría realizarse para determinar si esta crisis política y los acontecimientos relacionados tuvieron impactos a corto o largo plazo, teniendo en cuenta que los ataques terroristas suelen tener efectos a corto plazo, mientras que la inestabilidad política podría llevar a una alteración de la imagen del destino (Araña y León, 2008; Saha y Yap, 2014).

\section{Bibliografía}

Altindag, Duha T. (2014). «Crime and International Tourism». Journal of Labor Research, 35(1): 1-14. doi: 10.1007/s12122-014-9174-8

Angelopoulou, Alexia y Roeder, Bernd (2016). Greek Tourism Still Affected by Refugee Crisis. Disponible en: http://www.iol.co.za/travel/world/europe/ greek-tourism-still-affected-by-refugee-crisis2003282 , acceso el 30 de junio de 2019.

Ansani, Andrea y Daniele, Vittorio (2012). «About a Revolution: The Economic Motivations of the Arab Spring". International Journal of Development and Conflict, 2(3): 1-25. doi: 10.1142/ S2010269012500135

Araña, Jorge E. y León, Carmelo J. (2008). «The Impact of Terrorism on Tourism Demand». Annals of Tourism Research, 35(2): 299-315. doi: 10.1016/j.annals.2007.08.003

Ardıç, Nurullah (2012). "Understanding the «Arab Spring»: Justice, Dignity, Religion and International Politics». Afro Eurasian Studies, 1(1): 8-52.
Avraham, Eli (2015). «Destination Image Repair during Crisis: Attracting Tourism during the Arab Spring Uprisings». Tourism Management, 47: 224-232. doi.org/10.1016/j.tourman.2014.10.003

Ballard, Chet y Prine, Rudy (2002). «Citizen Perceptions of Community Policing: Comparing Internet and Mail Survey Responses". Social Science Computer Review, 20(4): 485-493. doi: 10.1177/089443902237324

Butler, Richard y Suntikul, Wantanee (eds.) (2013). Tourism and War. London y New York: Routledge.

Carr, Neil (2001). «An Exploratory Study of Gendered Differences in Young Tourists Perception of Danger within London". Tourism Management, 22(5): 565-570. Disponible en: http:// dx.doi.org/10.1016/S0261-5177(01)00014-0

Cohen, Erik (1972). «Toward a Sociology of International Tourism». Social Research, 39(1): 164182. Disponible en: http://www.jstor.org/ stable/40970087, acceso el 17 de febrero de 2020.

Costello, Matthew, J.; Jenkins, Craig J. y Aly, Hassan (2015). «Bread, Justice, or Opportunity? The Determinants of the Arab Awakening Protests". World Development, 67: 90-100. doi: 10.1016/j. worlddev.2014.10.002

Croatian Bureau of Statistics (2011-2017). Tourist Arrivals and Nights. Disponible en: https://www. dzs.hr/Hrv_Eng/publication/, acceso el 17 de febrero de 2020.

Crompton, John L. (1979). «Motivations for Pleasure Vacation". Annals of Tourism Research, 6(4): 408-424. doi: 10.1016/0160-7383(79)90004-5

D'Alema, Francesco (2017). The Evolution of Turkey's Syria Policy. Disponible en: http://www.iai. it/sites/default/files/iaiwp1728.pdf, acceso el 3 de mayo de 2019.

Dalmasso, Emanuela y Cavatorta, Francesco (2013). «Democracy, Civil Liberties and the Role of Religion after the Arab Awakening: Constitutional Reforms in Tunisia and Morocco". Mediterranean Politics, 18(2): 225-241. doi: 10.1080/13629395.2013.799341

Desrues, Thierry (2009). «El islamismo en el mundo árabe. Interpretaciones de algunas trayectorias políticas». Revista Internacional de Sociología, 67(1): 9-28. doi: 10.3989/ris.2009.i1.120

Dolnicar, Sara; Laesser, Christian y Matus, Katrina (2009). "Online Versus Paper: Format Effects in Tourism Surveys". Journal of Travel Research, 47(3): 295-316. doi: 10.1177/0047287508326506 
Drakos, Konstantinos y Kutan, Ali M. (2003). «Regional Effects of Terrorism on Tourism in Three Mediterranean Countries". Journal of Conflict Resolution, 47(5): 621-641. doi: 10.1177/0022002703258198

Elsrud, Torun (2001). «Risk Creation in Traveling: Backpacker Adventure Narration". Annals of Tourism Research, 28(3): 597-617. Disponible en: http://dx.doi.org/10.1016/S0160-7383(00)00061-X

Enloe, Cynthia (2014). «Gender Makes The World Go Around». En: Enloe, C. (ed.). Bananas, Beaches and Bases: Making Feminist Sense of International Politics. Berkeley, California: University of California Press, pp. 1-36.

Fletcher, John y Morakabati, Yeganeh (2008). «Tourism Activity, Terrorism and Political Instability within the Commonwealth: The Cases of Fiji and Kenya». International Journal of Tourism Research, 10(6): 537-556. doi: 10.1002/jtr.69

Fourie, Johan; Rosselló-Nadal, Jaume y Santana-Gallego, María (2020). «Fatal Attraction: How Security Threats Hurt Tourism". Journal of Travel Research, 59(2): 209-219. doi: 10.1177/0047287519826208

FRONTEX (2012-2016). Risk Analysis. Disponible en: http://frontex.europa.eu/publications, acceso el 13 de abril de 2019.

Fuchs, Galia y Reichel, Arie (2011). «An Exploratory Inquiry into Destination Risk Perceptions and Risk Reduction Strategies of First Time vs. Repeat Visitors to a Highly Volatile Destination». Tourism Management, 32(2): 266-276. doi: 10.1016/j.tourman.2010.01.012

Gibson, Heather y Yiannakis, Andrew (2002). «Tourist Roles: Needs and the Adult Life Course». Annals of Tourism Research, 29(2): 358-383. doi: 10.1016/S0160-7383(01)00037-8

Ghaderi, Zahed; Saboori, Behnaz y Khoshkam, Mana (2017). «Does Security Matter in Tourism Demand?». Current Issues in Tourism, 20(6): 552-565. doi: 10.1080/13683500.2016.1161603

Grandits, Hannes y Taylor, Karin (eds.) (2010). Yugoslavia's Sunny Side: A History of Tourism in Socialism (1950s-1980s). Budapest: CEU Press.

Gustafson, Per E. (1998). «Gender Differences in Risk Perception: Theoretical and Methodological Perspectives». Risk Analysis, 18(6): 805-811. http:// dx.doi.org/10.1111/j.1539-6924.1998.tb01123.x

Hawes, Douglass K. (1988). "Travel-Related Lifestyle Profiles of Older Women". Journal of Travel Research, 27(2): 22-32. doi: 10.1177/004728758802700204
Hellenic Statistical Authority (2011-2016). Arrivals of Non-Residents from Abroad, By Country of Residence. Disponible en: https://www.statistics.gr/ en/statistics/-/publication/STO04/2010-Q1, acceso el 17 de febrero de 2020.

Hoffman, Michael y Jamal, Amaney (2012). «The Youth and the Arab Spring: Cohort Differences and Similarities». Middle East Law and Governance, 4(1): 168-188. doi: 10.1163/187633712X632399

Iso-Ahola, Seppo E. (1982). «Toward a Social Psychological Theory of Tourism Motivation: A Rejoinder». Annals of Tourism Research, 9(2): 256262. doi: 10.1016/0160-7383(82)90049-4

Iso-Ahola, Seppo E. (1983). «Towards a Social Psychology of Recreational Travel». Leisure Studies, 2(1): 45-56. doi: 10.1080/02614368300390041

Kobašić, Antun (1987). Turizam u Jugoslaviji -razvoj, stanje i perspective / Tourism in Yugoslavia- Development, Situation and Perspectives. Zagreb: Inormator.

Kôvári, István y Zimányi, Krisztina (2011). "Safety and Security in the Age of Global Tourism (The Changing Role and Conception of Safety and Security in Tourism)". APSTRACT: Applied Studies in Agribusiness and Commerce, 5(3-4): 59-62.

Law, Rob (2006). «The Perceived Impact of Risks on Travel Decisions». International Journal of Tourism Research, 8(4): 289-300. doi: 10.1002/jtr.576

Lepp, Andrew y Gibson, Heather (2003). «Tourist Roles, Perceived Risk and International Tourism". Annals of Tourism Research, 30(3): 606-624. doi: 10.1016/S0160-7383(03)00024-0

Lim, Christine (1999). "A Meta-Analytic Review of International Tourism Demand". Journal of Travel Research, 37(3): 273-284. doi: 10.1177/004728759903700309

Llorca-Vivero, Rafael (2008). "Terrorism and International Tourism: New Evidence». Defence and Peace Economics, 19(2): 169-188. doi: 10.1080/10242690701453917

Mansfeld, Yoel y Pizam, Abraham (eds.) (2006). Tourism, Security and Safety - From Theory to Practice. London y New York: Routledge.

Mansfeld, Yoel y Winckler, Onn (2015). "Can This Be Spring? Assessing the Impact of the "Arab Spring" on the Arab Tourism Industry". Tourism - An International Interdisciplinary Journal, 63(2): 205-223. Disponible en: https:// hrcak.srce.hr/index.php?show=clanak\&id_clanak jezik=205893\&lang=en, acceso el 17 de febrero de 2020. 
Marion, Karl (2018). «Risk and Uncertainty in Travel Decision-Making: Tourist and Destination Perspective». Journal of Travel Research, 57(1): 129146. doi: $10.1177 / 0047287516678337$

Mayo, Edvard J. y Jarvis, Lance P. (1981). The Psychology of Leisure Travel: Effective Marketing and Selling of Travel Services. Boston: CBI Publishing.

Micić Jasna; Denda, Stefan y Popescu, Marius (2019). "The Significance of the Risk Related Challenges in Tourist Destination Choice». Journal of the Geographical Institute "Jovan Cvijić» SASA, 69(1): 39-52. doi: 10.2298/IJGI1901039M

Middleton, Victor T. C.; Fyall, Alan; Morgan, Michael y Ranchhod, Ashok (2009). Marketing in Travel and Tourism. Oxford: Butterworth-Heinemann. (4. ${ }^{a}$ ed.).

Morakabati, Yeganeh (2007). Tourism, Travel Risk and Travel Risk Perceptions: A Study of Travel Risk Perceptions and the Effects of Incidents on Tourism. Bournemouth: Bournemouth University. [Tesis doctoral]. Disponible en: https://ethos. bl.uk/OrderDetails.do?uin=uk.bl.ethos.486294, acceso el 8 de febrero de 2020.

Neumayer, Eric (2004). «The Impact of Political Violence on Tourism: Dynamic Cross-National Estimation». Journal of Conflict Resolution, 48(2): 259-281. doi: 10.1177/0022002703262358

Neumayer, Eric y Plümper, Thomas (2016). «Spatial Spill-Overs from Terrorism on Tourism: Western Victims in Islamic Destination Countries». Public Choice, 169(3): 195-206. doi: 10.1007/s11127016-0359-y

Niemi, Richard G.; Portney, Kent E. y King, David (2008). Sampling Young Adults: The Effects of Survey Mode and Sampling Method on Inferences about Political Behavior. Disponible en: http://citation.allacademic.com/meta/p_mla apa_research_citation/2/7/9/9/5/p279955_i ndex. html, acceso el 20 de abril de 2019.

Öniş, Ziya (2014). «Turkey and the Arab Revolutions: Boundaries of Regional Power Influence in a Turbulent Middle East». Mediterranean Politics, 19(2): 203-219. doi: 10.1080/13629395.2013.868392

Park, Kwangsoo y Reisinger, Yvette (2010). «Differences in the Perceived Influence of Natural Disasters and Travel Risk on International Travel». Tourism Geographies, 12(1): 1-24. doi: $10.1080 / 14616680903493621$

Perles-Ribes, José Francisco; Ramón-Rodríguez, Ana Belén; Moreno-Izquierdo, Luis y Torregrosa Martí, María Teresa (2018). «Winners and Losers in the Arab Uprisings: A Mediterranean Tourism
Perspective». Current Issues in Tourism, 21(16): 1810-1829. doi: 10.1080/13683500.2016.1225697

Pizam, Abraham; Reichel, Arie; Boemmel, Hermann van y Jeong, Gang-Hoan (2004). «The Relationship between Risk-Taking, Sensation-Seeking, and the Tourist Behavior of Young Adults: A Cross-Cultural Study". Journal of Travel Research, 42(3): 251-260. doi: $10.1177 / 0047287503258837$

Plog, Stanley C. (1974). «Why Destination Areas Rise and Fall in Popularity". Cornell Hotel and Restaurant Quarterly, 14(4): 55-58. doi: 10.1177/001088047401400409

Reisinger, Yvette y Mavondo, Felix (2006). «Cultural Differences in Travel Risk Perception». Journal of Travel \& Tourism Marketing, 20(1): 13-31. doi: 10.1300/J073v20n01_02

Roehl, Wesley S. y Fesenmaier, Daniel R. (1992). «Risk Perceptions and Pleasure Travel: An Exploratory Analysis». Journal of Travel Research, 30(4): 17-26. doi: 10.1177/004728759203000403

Saha, Shrabani y Yap, Ghialy (2014). «The Moderation Effects of Political Instability and Terrorism on Tourism Development: A Cross-Country Panel Analysis». Journal of Travel Research, 53(4): 509521. doi: $10.1177 / 0047287513496472$

Salih, Kamal Eldin Osman (2013). «The Roots and Causes of the 2011 Arab Uprisings». Arab Studies Quarterly, 35(2): 184-206. doi: 10.13169/ arabstudquar.35.2.0184

Sönmez, Sevil F. y Graefe, Alan R. (1998). «Influence of Terrorism Risk on Foreign Tourism Decisions". Annals of Tourism Research, 25(1): 112-144. doi: 10.1016/S0160-7383(97)00072-8

Statistical Office of Montenegro (2011-2016). Statistical Yearbook. Disponible en: http://monstat. org/eng/publikacije_page.php?id=196, acceso el 17 de febrero de 2020.

Statistical Office of the Republic of Serbia (20112016). Statistical Yearbooks of the Republic of Serbia. Disponible en: http://www.stat.gov.rs/ publikacije/, acceso el 20 de abril de 2019.

Steiner, Christian (2007). «Political Instability, Transnational Tourist Companies and Destination Recovery in the Middle East after 9/11». Tourism and Hospitality Planning \& Development, 4(3): 169-190. doi: 10.1080/14790530701733421

Tarlow, Peter E. (2006). A Social Theory of Terrorism and Tourism. En: Mansfred, Y. y Pizam, A. (eds.). Tourism Security and Safety - From Theory to Practice. London y New York: Routledge, pp. 33-47. 
Tarlow, Peter E. (2014). Tourism Security - Strategies for Effectively Managing Travel Risk and Safety. Amsterdam: Butterworth-Heinemann.

Teoman, Denis Can (2017). «Terrorism and Tourism in Europe, New Partners». European Journal of Geography, 8(2): 132-142.

Theocharous, Antonis L.; Zopiatis, Anastasios; Lambertides, Neophytos; Savva, Christos S. y Mansfeld, Yoel (2018). «Tourism, Instability and Regional Interdependency: Evidence from the Eastern-Mediterranean". Defence and Peace Economics, 0(0): 1-24. doi: 10.1080/10242694.2018.1501531

Tomazos, Konstantinos (2017). Egypt's Tourism Industry and the Arab Spring. En: Butler, R. y Suntikul, W. (eds.). Tourism and Political Change. Oxford: Goodfellow Publishers. (2. ${ }^{\mathrm{a}}$ ed.).

UNWTO (2011-2017). UNWTO Tourism Highlights, 2011-2017 Editions. Disponible en: ht-
tps://www.eunwto.org/action/showPublications? category $=10.1555 \% 2 \mathrm{Fcateg}$ ory .4000 0037 \&pageSize $=20 \&$ startPage $=2$, acceso el 20 de mayo de 2019.

Vanhove, Norbert (2011). The Economics of Tourism Destinations. London: Elsevier.

World Economic Forum (2011-2017). The Travel \& Tourism Competitiveness Report. Disponible en: http://www.weforum.org/reports, acceso el 20 de abril de 2019.

Wearing, Betsy y Wearing, Stephen (1996). «Refocusing the Tourist Experience: The Flaneur and the Choraster». Leisure Studies, 15(4): 229-243.

Yılmaz, Bahri (2012). "Turkey and the Arab Spring: The Revolutions in Turkey's Near- Abroad". En: Calleya, S. y Wohlfeld, M. (eds.). Change and Opportunities in the Emerging Mediterranean. Malta: Mediterranean Academy of Diplomatic Studies, pp. 349-369.

RECEPCIÓN: 18/07/2019

REVISIÓN: $14 / 01 / 2020$

APROBACIÓN: 13/05/2020 


\title{
The Impact of Contemporary Politically Induced Security Crisis on Tourist Behaviour: The perception of the Serbian Younger Adult Population
}

\author{
El impacto de la crisis de seguridad contemporánea inducida políticamente en \\ el comportamiento de los turistas: la percepción de la población joven serbia
}

\section{Stefan Denda, Jasna Micić and Aleksandra Terzić}

\section{Key words \\ Tourists' Attitudes \\ - Tourism Destination \\ Choice \\ - Young Adult and \\ Young Middle-Aged \\ - Mediterranean \\ Countries \\ - Arab Uprisings \\ - Security Risks \\ - Serbian \\ - Political Violence and \\ Tourist Impacts}

\section{Palabras clave}

Actitudes de turistas

- Elección de destino turístico

- Jóvenes

- Países mediterráneos

- Primavera Árabe

- Riesgo de seguridad

- Serbia

- Violencia política e impactos turísticos

\begin{abstract}
The study examines how tourists perceive the security crisis in the MENA region and to what extent it affects their travel decision process. It is based on a survey conducted among Serbian young adult and younger middle-aged residents. The survey tracked the demographic and socioeconomic characteristics of the respondents, their travel habits and attitudes towards the security situation in the Mediterranean. The research shows that the target population is well-aware of the current security situation. The non-parametric statistical tests conducted reveal differences by gender in risk perception. Even though a relocation of tourism demand in Serbia has occurred because of the security crisis, the study findings indicate that the security situation is not a decisive factor in destination selection.
\end{abstract}

\section{Resumen}

La investigación tiene por objetivo determinar cómo los turistas perciben la crisis de seguridad en la región MENA y en qué medida afecta al proceso de decisión de viaje. Se realizó entre los residentes jóvenes y de mediana edad serbios. La encuesta hizo un seguimiento de las características demográficas y socioeconómicas de los encuestados, sus hábitos de viaje y actitudes hacia la situación de seguridad en el Mediterráneo. El público objetivo es muy consciente de la situación de seguridad. Las pruebas estadísticas no paramétricas revelaron las diferencias de género en la percepción del riesgo. Aunque la reubicación de la demanda turística de Serbia se ha producido como consecuencia de la crisis, la investigación muestra que la situación de seguridad no es un factor decisivo en la selección del destino.

\section{Citation}

Denda, Stefan; Micić, Jasna and Terzić, Aleksandra (2021). "The Impact of Contemporary Politically Induced Security Crisis on Tourist Behaviour: The perception of the Serbian Younger Adult Population". Revista Española de Investigaciones Sociológicas, 174: 69-88. (http://dx.doi.org/10.5477/cis/ reis.174.69)

Stefan Denda: Geografski Institut Jovan Cvijic Srpska Akademija Nauka i Umetnosti (Serbia) | s.denda@gi.sanu.ac.rs Jasna Micić: Geografski Institut Jovan Cvijic Srpska Akademija Nauka i Umetnosti (Serbia) | j.micic@gi.sanu.ac.rs Aleksandra Terzić: Geografski Institut Jovan Cvijic Srpska Akademija Nauka i Umetnosti (Serbia) | a.terzic@gi.sanu.ac.rs 


\section{INTRODUCTION}

The tourism industry has undergone continual growth over recent decades (Middleton et al., 2009). As a result, it has become an important source of revenue for many countries all over the world. However, despite this positive trend, periods of decline have also occurred: 1967/1968, 1973/1974 (economic recession), the mid-1980s (Chernobyl incident; Libyan bombing), early 1990s (the Gulf War and the Wars in Yugoslavia), 2000s (terrorist attacks in the USA, Madrid, London and Bali; the Afghanistan War and the Iraq War; SARS; the Asian tsunami, and Icelandic ash), and the global financial crisis in 2008/2009 (Vanhove, 2011). Over time, tourism has changed both in quantitative and qualitative terms, as factors that influence tourists' behaviour have become more complex. There are many tourism motivation theories (Cohen, 1972; Crompton, 1979; Iso-Ahola, 1982, 1983; Mayo and Jarvis, 1981; Plog, 1974) that have tried to systematize chaotic travel decision-making processes. As Lim (1999) argued, income, transportation costs and tourism prices are the most commonly used variables in empirical studies of tourism demand. Even though it is understood that certain events have an impact on the sense of perceived security at global and regional levels (e.g., terrorism, natural disasters, migrations, contagious diseases, etc.) (Kôvári and Zimányi, 2011), the relationship between tourism demand and safety and security in tourism destinations has been given limited attention by both researchers and policymakers (Fourie, Roselló-Nadal and Santana-Gallego, 2020; Ghaderi, Saboori and Khoshkam, 2017). The terrorist attack on the Twin Towers in New York (2001) changed potential tourists' perceptions of global security, as travellers are now aware of this relationship (Lepp and Gibson, 2003).
Since the 1990s, terrorist attacks targeting tourists have been recorded at tourist destinations with majority Islamic populations (e.g., Egypt, Tunisia and Turkey). However, over the past several years, tourists at Western destinations (France, Germany, Spain and Belgium) have also become attack targets. This phenomenon is associated with the period of political riots in Middle Eastern and North African countries commonly referred to as the "Arab Spring". Since the end of 2010, this unrest has had a great political and socioeconomic impact in the Mediterranean region. As a direct consequence of this instability, Europe has experienced its worst migration and security crisis since World War II. Additionally, all these events have had significant impact on the development of the tourism industry, especially in the Middle East and North Africa (MENA) region.

As Mansfeld and Winckler (2015) stated, the Arab Spring crisis in tourism is different from other crises due to its duration and the nature of the new regimes. Avraham (2015) revealed negative effects from the crisis on the tourism industry in the MENA region; however, there is still a lack of empirical studies that explain the impacts of this revolution (Tomazos, 2017; Theocharous et al., 2018).

The relationship between safety and security and tourism demand has not been well researched; this study aims to fill this gap in the current social and tourism literature. The impact of the Arab Spring crisis on tourism demand is the focus of this study due to its far reaching effects and its duration, along with the existence of a general impression of insecurity in the MENA region, even when there is no official war (Butler and Suntikul, 2013). The research question we attempt to answer is whether and to what extent this crisis and accompanying events have affected tourist demand to visit the MENA region. 


\section{Arab Spring as a contemporary SECURITY CHALLENGE}

The Arab uprisings started on December 17th, 2010, when the street vendor Mohammed Bouazizi set himself on fire after the confiscation of his goods by the police in Tunisia (Ansani and Daniele, 2012). This event was known as the "Jasmine Revolution". From January to November 2011, the initial uprising in Tunisia was followed by a series of massive violent events in countries across the MENA region (Tunisia, Egypt, Libya, Morocco, Yemen, Jordan, Bahrain, Syria, and others). The "Arab street" and the "Arab city" (Tahrir Square in Cairo, Martyrs' Square in Tripoli, etc.) were the places of the "revolutionary occupation" (Ardıç, 2012). As urban youth constituted a large proportion of the population, they played a tremendous role in the revolution. This cohort (15-29 years of age) was less religious and thus less supportive of political Islam. In addition, they were more educated, but many of them were unemployed (accounting for $25 \%$ of the total unemployed population in Tunisia and Egypt), while almost $50 \%$ were women. Their dissatisfaction with the political and economic situation was enormous (Hoffman and Jamal, 2012).

Urban youth used information and communication technologies as the main instrument to organise mass gatherings and spread slogans (Costello, Jenkins and Aly, 2015). The unrest was supported by external powers, such as the European Union, the US, Turkey and NATO, due to their geostrategic interests in the region, especially after September 11th, 2001. Turkey's role in particular was important for many reasons. For Islamic states, Turkey was an example of capitalism and Islam working together (YIlmaz, 2012). Furthermore, for Turkey, its Middle Eastern neighbours were the gateway to the Arab world (economically and politically) (Öniş, 2014). Finally, Turkey conducted an audit of its strategic priorities and undertook to co-operate with Russia towards resolving the Syrian conflict. As a result, cooperation with Western partners (the US and the EU) declined after the failed coup of 2016 in Turkey (D'Alema, 2017). All these pressures fostered political changes in these countries. In Tunisia and Egypt, a new political order was established; Morocco and Jordan made constitutional changes, while a new president was elected in Jordan. At the same time, Libya was completely fragmented, while Syria experienced a civil war that continues today (Salih, 2013). These developments led to the strengthening of Islamism and the emergence of political movements (such as the Muslim Brotherhood and the Al-Nour Party in Egypt) and militant groups (e.g., the Islamic State of Iraq and Syria, Ansar al-Sharia in Libya and the Al-Nusra Front in Syria) (Dalmasso and Cavatorta, 2013).

These conflicts caused massive migrations towards Europe. According to EU agency reports, four major migratory routes (Central, Western, and Eastern Mediterranean, and Western Balkan routes) and three additional routes (Circular Route from Albania to Greece, Eastern Borders, and the West African Route) have been identified. Bearing in mind this route classification, the increasing number of migrants in Greece (Eastern Route) had a direct effect on the Western Balkan Route (FRONTEX, 2012-2016). Migrants tried to continue their journey to Hungary and Croatia via North Macedonia and Serbia. This route became popular in 2012 due to the Schengen Agreement, which abolished visas for the former Yugoslav countries and Albania. Since 2013, the number of border crossings has steadily grown, reaching 764,000 in 2015 (14-16 times more than in 2014) (FRONTEX, 2012-2016). 


\section{THE IMPACT OF SECURITY RISKS ON MediterRanean tourism}

Globally, the conflicts have had a tremendous impact on all aspects of international relations, including tourism. More than $1 / 3$ of European tourist turnover was accounted for by the Southern/Mediterranean subregion (18-19\% of world tourist arrivals). These countries (France, Spain, Italy, Turkey, Greece and Croatia) had variable average annual growth rates in arrivals from $2 \%$ to $23 \%$. Individually, Spain and Greece witnessed the greatest proportional increase in visitors (2014) and France a small decline (2016) due to security incidents in Paris and Nice (UNWTO, 2011-2017). In 2011 and
2015, the number of arrivals in the North African MENA region decreased (by $3 \%$ ) due to geopolitical, security and economic challenges. These problems had a huge impact in the North African Mediterranean region (a decrease of 6-12\%), especially in Tunisia $(-31 \%$ in 2011$)$ due to political changes (UNWTO, 2011-2017) (see Table 1). Furthermore, in the Middle Eastern region, negative growth in tourism was recorded $(-8 \%)$ due to security risks in 2011. At the destination level, the greatest turbulence was experienced by Egypt $(-31 \%$ in 2011 and 2014; $-42 \%$ in 2016) due to various incidents (political tensions, the military coup, Syrian civil war, etc.) (UNWTO, 2011-2017).

TABLE 1. International tourist arrivals (2010-2016) in selected countries (in millions)

\begin{tabular}{lrrrrrrr}
\hline Country & $\mathbf{2 0 1 0}$ & $\mathbf{2 0 1 1}$ & $\mathbf{2 0 1 2}$ & $\mathbf{2 0 1 3}$ & $\mathbf{2 0 1 4}$ & $\mathbf{2 0 1 5}$ & $\mathbf{2 0 1 6}$ \\
\hline Spain & 52.7 & 56.7 & 57.7 & 60.6 & 65.0 & 68.2 & 75.6 \\
France & 76.8 & $\mathbf{7 9 . 6}$ & $\mathbf{8 3 . 0}$ & $\mathbf{8 3 . 6}$ & $\mathbf{8 3 . 7}$ & $\mathbf{8 3 . 7}$ & $\mathbf{8 2 . 6}$ \\
Italy & 43.6 & 46.1 & 46.4 & $\mathbf{4 7 . 7}$ & $\mathbf{4 8 . 6}$ & 50.7 & 52.4 \\
Greece & 15.0 & 16.4 & 15.5 & 17.9 & 22.0 & 23.6 & 24.8 \\
Croatia & 9.1 & 9.9 & 10.3 & 11.0 & 11.6 & 12.7 & 13.8 \\
Montenegro & 0.5 & 1.2 & 1.3 & 1.3 & 1.3 & 1.6 & 1.7 \\
Morocco & 9.3 & 9.3 & 9.4 & 10.0 & 10.3 & 10.2 & 10.3 \\
Tunisia & 6.9 & 4.8 & 5.9 & 6.3 & 6.1 & 5.4 & 5.7 \\
Egypt & 14.1 & 9.5 & 11.2 & 9.2 & 9.6 & 9.1 & 5.3 \\
Turkey & 27.0 & 29.3 & 35.7 & 37.8 & 39.8 & 39.5 & 25.3 \\
\hline
\end{tabular}

Source: UNWTO Tourism Highlights, 2011-2017.

It is noteworthy that the tourism industry is a strategic sector for most Mediterranean countries, and especially Tunisia, Egypt, Morocco and Turkey. It is a "backbone" of their economies (Perles-Ribes et al., 2018). According to the World Economic Forum (20112017), tourism accounted for $5-10 \%$ of the GDP in these countries. At the same time, this sector provided $3-10 \%$ of total jobs. With the crisis, the economic performance of the travel and tourism industry was reduced, particularly in the North African and Middle Eastern countries of the Mediterranean. Tourism revenues decreased drastically. Observed at the country level, the largest decline in revenues was recorded in Egypt (>400\%), Tunisia (>200\%), 
and Turkey (160\%). In these regions, tourism depended greatly on European tourists who accounted for over $60 \%$ of the total demand. Thus, once again, the effects of the spread of political instability from neighbouring countries are highlighted (Steiner, 2007).

\section{THE RELATIONSHIP BETWEEN SECURITY ISSUES AND DESTINATION CHOICES}

In the tourism industry, the terms security and safety have been often used interchangeably, although in other disciplines a clear distinction is made between them. The explanation could be found in the high sensitivity of tourism to any type of disruption (Tarlow, 2014). During the process of choosing a tourist destination, potential tourists assess destination alternatives by comparing the costs and benefits of the destination. Sönmez and Graefe (1998) proposed an international travel decision-making framework. They categorized factors that influence destination choice into three categories: external factors (mass media, government travel advisories and social interactions), internal factors (travel experience, attitudes and perceptions of safety) and demographic factors. Additionally, Roehl and Fesenmaier (1992), as well Sönmez and Graeffe (1998) evaluated nine types of risks associated with travel: financial, health, physical, social, psychological and temporal risk, satisfaction, political instability and terrorism.

In recent decades, terrorism has been strongly associated with the tourism industry. Since 2001 and the terrorist attacks in the USA, the concept of tourism has changed. Travel and tourism officials have become involved in national and international security issues. As Tarlow (2014: 98) argues, "tourism has been a magnet for terrorist attacks". There are several reasons why tourism and visitors are attractive to terrorists. Tourism provides terrorists' opportunities for mass casualties at iconic locations (Tarlow, 2014). Tourists are vulnerable and easy to attack.
They are so-called "soft" targets because during holidays, they tend to relax and they are less cautious about their surroundings (Tarlow, 2006). In many cases, targets are "Western" tourists. The "West" is usually perceived as supporting the weak governments of Islamic countries and seeking to stop radical groups struggling to gain dominance. In addition, as mass media are mostly owned by Western corporations, "killing Western citizens is much more likely to attract global media attention" (Neumayer and Plümper, 2016). Furthermore, the lifestyle and behaviour of tourists (the way they dress, their alcohol consumption, gambling, etc.) have been condemned by terrorists. Nowadays, a smaller number of places can be considered safe, which shows that terrorists have already succeeded in creating a climate of fear around the world (Sönmez and Graefe, 1998).

Empirical studies conducted so far have shown that security issues and their impact on tourism demand patterns depend on various factors. In the first place, these are sociodemographic factors, such as gender (Pizam et al., 2004), age (Marion, 2018; Reisinger and Mavondo, 2006; Sönmez and Graefe, 1998) and education (Marion, 2018; Park and Reisinger, 2010; Sönmez and Graefe, 1998). In addition, risk perception is also impacted by travelling with small children (Sönmez and Graefe, 1998), cultural background (Lepp and Gibson, 2003), personality type (risk-averse individuals and risk seekers), previous travel experience and nationality (especially among Western visitors) (Fuchs and Reichel, 2011). However, there are contradictory findings regarding certain factors. For example, concerning the significance of gender-related differences on perceived travel risks, some authors found that gender does not influence risk perception (Carr, 2001; Sönmez and Graefe, 1998), while other studies pointed to the essential role of this factor (Elsrud, 2001; Enloe 2014; Gustafson, 1998; Hawes, 1988; Wearing and Wearing, 1996). 
Many researchers agree that safety and security are significant factors determining tourism demand, and a precondition for the success of international tourism (Ghaderi, Saboori and Khoshkam, 2017; Fletcher and Morakabati, 2008; Mansfeld and Pizam, 2006). If the potential risks of becoming a victim of terrorism or crime outweigh benefits, tourists are likely to cancel their trips despite destination loyalty (Altindag, 2014; Law, 2006).

Based on the current state and findings of recent studies, the main aim and specific goals of this study are to clarify the impact of certain factors on individual security and international tourism demand. The overall aim of this study is to determine whether and to what extent the political and security crisis induced by events in the MENA region affected Serbian young adult and younger middle-aged tourists' behaviour. Following this general question, five specific hypotheses have been defined:

1. The perception of safety and security depends on gender.

2. Safety and security concerns depend on age and income.

3. Travel behaviour of the Serbian young adult and younger middle-aged population has changed during the crisis.

4. The media image of the current security situation increases potential tourists' sense of insecurity.

5. The perceived safety and security of the destination is a decisive factor in destination decision-making.

\section{Methods ANd data COllection}

The Mediterranean region has been among most important global tourism destinations, with an enormous number of tourist arrivals and overnight stays. However, in recent years, it has faced numerous security prob- lems caused by the Arab Spring crisis. The growth in migrations and the creation of the important Western Balkan migration route, which passes through Serbia, was the main reason why this area was chosen for our study. Additionally, Mediterranean countries have traditionally been the main vacation choice for Serbian tourists, so we wanted to ascertain whether and to what extent politically-induced security issues have influenced changes in tourist behaviour.

This study focuses on a specific segment of the population, young adult and younger middle-aged tourists (age group: 19-39), who account for $26.6 \%$ of the Serbian population (13.5\% male, $13.1 \%$ female). Several studies have found that safety concerns grow with age (Gibson and Yiannakis, 2002; Sönmez and Graefe, 1998), so we wanted to examine how this group perceives risks and responds to security and safety issues and to determine if some different and specific principles apply to this group.

The research was conducted using a self-administrated open online survey as a research technique. This way of collecting data in tourism studies has proven to be appropriate because it facilitates obtaining more complete and quality responses (Ballard and Prine, 2002; Dolnicar, Laesser and Matus, 2009). It is particularly useful when it comes to this specific age group of respondents, which is difficult to reach with other methods (e.g., face-to-face interviews, telephone surveys, etc.) (Niemi, Portney and King, 2008).

Data were collected among Serbian young adult and younger middle-aged residents during June and July 2016. A sample of 301 respondents was collected, while 249 responses were considered valid for the study. Some of the respondents were excluded from further analysis because they did not satisfy the age group requirement, or due to certain technical reasons (incomplete and contradictory answers, lack of data, etc.) (Table 2). 
TABLE 2. Technical sheet

\begin{tabular}{lc}
\hline Questionnaires collected & 301 \\
Questionnaires valid & 249 \\
Response rate & $82.72 \%$ \\
Confidence level & $95 \%$ \\
Type of survey & self-administrated \\
Survey completed & July 2016 \\
Statistical procedures & SPSS statistics \\
\hline
\end{tabular}

Source: By authors.

The final questionnaire had three parts. The first part was focused on the socioeconomic and demographic characteristics of the respondents, aligned with the official Census methodology of Serbia (2011). The second part examined the existing travel habits of Serbian citizens. In the third part, the attitudes of tourists who spent their vacations in Mediterranean destinations affected by numerous security risks were examined. The questionnaire included several types of questions: open-ended, close-ended and Likert-scale questions. The values on the Likert-scale ranged from 1 (strongly disagree) to 5 (strongly agree). The Statistical Package for Social Sciences (version 21.0) was used for data processing. Finally, in the data analysis, two statistical tests for group comparison were used (Chi-square and Man-Whitney U test). Bearing in mind that prerequisites for Pearson's correlation were violated (normality, homoscedasticity and linearity), we applied a non-parametric correlation analysis.

\section{Results AND DISCUSSION}

\section{Sample description}

As mentioned, the results are based on a survey of 249 young adult and younger middle-aged Serbian tourists $(82.7 \%$ valid responses). A majority of the respondents were female $(66.7 \%)$. As far as the age structure is concerned, the respondents from the 19-29 group accounted for $69.1 \%$, while the group aged $30-39$ accounted for $30.9 \%$. This indicates that the younger population was more willing to participate in the research. Almost $80 \%$ of the respondents had higher education and $53 \%$ were employed. More than 20\% were without income and almost $30 \%$ had earnings ranging from 25,000 RSD a month (around 200 EUR) to 50,000 RSD (around 450 EUR). Almost $15 \%$ of the respondents did not want to answer the question regarding their monthly earnings because they considered it extremely personal (Table 3).

TABLE 3. Socio-demographic characteristics of the respondents

\begin{tabular}{|c|c|c|}
\hline Demographics & Frequencies & $\begin{array}{c}\text { Percentage } \\
(\%)\end{array}$ \\
\hline \multicolumn{3}{|l|}{ Gender } \\
\hline Female & 166 & 66.7 \\
\hline Male & 83 & 33.3 \\
\hline \multicolumn{3}{|l|}{ Age } \\
\hline $19-29$ & 172 & 69.1 \\
\hline $30-39$ & 77 & 30.9 \\
\hline \multicolumn{3}{|l|}{ Education level } \\
\hline High school & 53 & 21.3 \\
\hline Academic degree & 196 & 78.7 \\
\hline \multicolumn{3}{|l|}{ Employment status } \\
\hline Student & 91 & 36.5 \\
\hline Employed & 132 & 53.0 \\
\hline Unemployed & 26 & 10.5 \\
\hline \multicolumn{3}{|l|}{$\begin{array}{l}\text { Monthly income } \\
\text { (in Euros) }\end{array}$} \\
\hline Without income & 55 & 22.1 \\
\hline$<200$ & 28 & 11.2 \\
\hline $201-450$ & 68 & 27.3 \\
\hline $451-650$ & 31 & 12.4 \\
\hline$>650$ & 33 & 13.3 \\
\hline $\begin{array}{l}\text { I do not want to an- } \\
\text { swer this question }\end{array}$ & 34 & 13.7 \\
\hline TOTAL & 249 & 100.0 \\
\hline
\end{tabular}

Source: By authors. 


\section{The political challenges and tourists' attitudes}

The respondents were asked to express their attitudes towards statements related to current security issues and their impact on perceived risk in tourism destinations. They demonstrated a high level of knowledge of the key phenomena in this field. Almost $80 \%$ were familiar with the terms "Islamic State" and "Balkan Migration Route", while only $2.8 \%$ did not have enough information. In Table 4 the respondents' attitudes toward certain challenges are presented. Regarding the statement "ISIS operations disrupt the tourism industry of Mediterranean countries", a large majority of the respondents $(72.2 \%)$ expressed agreement. Additionally, there was a high rate of neutral responses (18.5\%), while a minority disagreed (strongly disagree $2.4 \%$ and disagree $6.8 \%$ ). The responses to the second statement "The Balkan migration route affects the decision-making process" were quite balanced: disagree $31.3 \%$, neutral $34.9 \%$, and agree $33.7 \%$. Regarding the statement, "The high migrant population density represents a risk for infectious diseases", more than 44\% agreed, along with neutral responses constituting almost $80 \%$ of responses. The fourth statement "New border management policy negatively influences destination choice", showed that potential tourists who agreed with the statement accounted for $48 \%$. The respondents expressed a prevailingly neutral attitude (53\%) towards the statement "The security services of the mentioned countries invest maximum efforts to improve security in destinations". Finally, almost $80 \%$ of the respondents agreed that "The media image of the current security situation increases the sense of insecurity of potential tourists".

TABLE 4. Frequencies of tourist attitudes (\%)

\begin{tabular}{|c|c|c|c|c|c|}
\hline Statements & 1 & 2 & 3 & 4 & 5 \\
\hline ISIS operations disrupt the tourism industry of Mediterranean countries. & 2.4 & 6.8 & 18.5 & 36.1 & 36.1 \\
\hline The Balkan migration route affects the decision-making process. & 10.8 & 20.5 & 34.9 & 21.3 & 12.4 \\
\hline $\begin{array}{l}\text { The high migrant population density represents a risk for infectious di- } \\
\text { seases. }\end{array}$ & 6.8 & 14.9 & 34.1 & 22.9 & 21.3 \\
\hline New border management policy negatively influences destination choice. & 11.2 & 17.7 & 23.3 & 29.7 & 18.1 \\
\hline $\begin{array}{l}\text { The security services of the mentioned countries invest maximum } \\
\text { efforts to improve security in destinations. }\end{array}$ & 6.0 & 13.3 & 53.0 & 21.7 & 6.0 \\
\hline $\begin{array}{l}\text { The media image of the current security situation increases the sense } \\
\text { of insecurity of potential tourists. }\end{array}$ & 1.6 & 4.8 & 16.5 & 43.4 & 33.7 \\
\hline
\end{tabular}

Note: 1 = strongly disagree, 2 = disagree, $3=$ neutral, $4=$ agree, $5=$ strongly agree.

Source: By authors.

To identify differences between men and women and among different age groups in attitudes towards the aforementioned statements, a chi-square test for independence was applied (Table 5). Bearing in mind that the study involved variables with more than two categories, Cramer's V coefficient was used to measure the strength of the relationship between the categorical variables. The Chi-square test revealed a significant relationship between the distribution of the respondents' attitudes and their gender concerning the statements "Impact of ISIS operations on tourism industry": $x^{2}$ $(4, \mathrm{n}=247)=12.91, p=0.01$, phi $=0.23$; "The high migrant population density rep- 
resents the risk for infectious diseases": $x^{2}$ $(4, \mathrm{n}=247)=10.50, p=0.03$, phi $=0.20$; and "New border management policy negatively influences destination choice": $x^{2}(4$, $\mathrm{n}=249)=13.21, p=0.01$, phi $=0.22$, with a probability of $5 \%(p \leq 0.05)$. In observed cases, Cramer's coefficient (V) shows moderate (acceptable) correlation strength between the variables. In the case of "The media image of the current security situation increases the sense of insecurity of potential tourists", the chi-square was $x^{2}$ $(4, \mathrm{n}=267)=9.28, p=0.05$, phi $=0.19$, but Cramer's coefficient suggests a weak correlation strength between the variables. Women provided more neutral and positive responses (agreement) than men. Among all the listed variables, a correlation was found between the age of the respondents and the statement "The security services of the mentioned countries invest maximum efforts to improve security in destinations": $X^{2}$ $(4, \mathrm{n}=267)=7.22, p=0.05$. Cramer's value (0.14) indicates a weak correlation. In this case, the percentage of those who disagreed with the statement was the greatest (19.3\%) in the younger group (19-29). Interestingly, the 30-39 group abstained the most in answering questions (53\% of neutral answers).

TABLE 5. The results of Chi-square test for independence

\begin{tabular}{lccc}
\hline \multicolumn{1}{c}{ Statements } & & $\begin{array}{c}\text { Chi-square } \\
\text { ( } \boldsymbol{p} \text { value) }\end{array}$ & $\begin{array}{c}\text { Cramer's V } \\
\text { Coefficient }\end{array}$ \\
\hline ISIS operations disrupt the tourism industry of Mediterranean & gender & $0.012^{\star \star}$ & 0.229 \\
countries. & age & 0.379 & 0.130 \\
The Balkan migration route affects the decision-making process. & gender & 0.095 & 0.178 \\
& age & 0.372 & 0.131 \\
The high migrant population density represents a risk for infec- & gender & $0.033^{\star \star}$ & 0.205 \\
tious diseases. & age & 0.227 & 0.151 \\
New border management policy negatively influences destina- & gender & $0.014^{\star}$ & 0.224 \\
tion choice. & age & 0.158 & 0.163 \\
The security services of the mentioned countries invest maxi- & gender & 0.696 & 0.094 \\
mum efforts to improve security in destinations. & age & $0.055^{\star *}$ & 0.170 \\
The media image of the current security situation increases the & gender & $0.054^{\star *}$ & 0.193 \\
sense of insecurity of potential tourists. & age & 0.690 & 0.095 \\
\hline
\end{tabular}

Note: ${ }^{\star} p \leq 0,01 ;{ }^{* \star} p \leq 0,05$.

Source: By authors.

The authors also compared medians between two independent groups (males and females) using a Man-Whitney $U$ test (Table 6). As a non-parametric version of the independent samples t-test, it evaluates whether the ranks of the two groups differ significantly. The results revealed that there was a significant difference between men and women regarding their attitudes toward the statements "The Balkan migration route affects the decision-making process" and "The media image of the current security situation increases the sense of insecurity of potential tourists". In the first statement $U=5695, z=-2.30, p=0.002$, with the same median for males and females $(M d m f=3, n m=83, n f=166)$. The significance for the second statement was 0.01 $(U=5553, z=-2.66, p=0.008)$ with $(M d=4$, $n=83$ ) for males and ( $M d=4, n=166)$ for females. Additionally, we calculated the effect size statistic value $(r)$ based on the $z$ value and the total number of cases. The $r$-value indicates a small effect size $(0.15$ for the first and 0.17 for the second statement). 
TABLE 6. The results of the Mann-Whitney $U$ test

\begin{tabular}{lccc}
\hline \multicolumn{1}{c}{ Statements } & U test & $\boldsymbol{z}$ & p \\
\hline $\begin{array}{l}\text { ISIS operations disrupt the tourism industry of Mediterranean coun- } \\
\text { tries. }\end{array}$ & 6,385 & -0.992 & 0.321 \\
The Balkan migration route affects the decision-making process. & 5,695 & -2.304 & $0.021^{\star *}$ \\
$\begin{array}{l}\text { The high migrant population density represents a risk for infectious } \\
\text { diseases. }\end{array}$ & 5,921 & -1.869 & 0.062 \\
$\begin{array}{l}\text { New border management policy negatively influences destination } \\
\text { choice. }\end{array}$ & 5,894 & -1.907 & 0.057 \\
$\begin{array}{l}\text { The security services of the mentioned countries invest maximum } \\
\text { efforts to improve security in destinations. }\end{array}$ & 6,325 & -1.150 \\
$\begin{array}{l}\text { The media image of the current security situation increases the sense } \\
\text { of insecurity of potential tourists. }\end{array}$ & 5,553 & -2.665 \\
\hline
\end{tabular}

Note: ${ }^{*} p \leq 0,01 ;{ }^{* \star} p \leq 0,05$.

Source: By authors.

TABLE 7. The results of correlation analysis

\begin{tabular}{lcccc}
\hline \multicolumn{1}{c}{ Statements } & & Gender & Age & $\begin{array}{c}\text { Monthly } \\
\text { incomes }\end{array}$ \\
\hline $\begin{array}{l}\text { ISIS operations disrupt the tourism industry of Mediterranean } \\
\text { countries. }\end{array}$ & rho & -0.063 & 0.048 & 0.021 \\
& $p$ & 0.322 & 0.048 & 0.742 \\
The Balkan migration route affects the decision-making process. & rho & -0.146 & 0.019 & -0.009 \\
& $p$ & $0.021^{* *}$ & 0.769 & 0.891 \\
The high migrant population density represents a risk for infec- & rho & -0.119 & -0.115 & -0.093 \\
tious diseases. & $p$ & 0.062 & 0.069 & 0.144 \\
$\begin{array}{l}\text { New border management policy negatively influences destina- } \\
\text { tion choice. }\end{array}$ & $p$ & -0.121 & -0.137 & -0.035 \\
$\begin{array}{l}\text { The security services of the mentioned countries invest maximum } \\
\text { efforts to improve security in destinations. }\end{array}$ & $p$ & -0.056 & $0.030^{* *}$ & 0.588 \\
$\begin{array}{l}\text { The media image of the current security situation increases the } \\
\text { sense of insecurity of potential tourists. }\end{array}$ & $p$ & -0.251 & 0.082 & 0.016 \\
\hline
\end{tabular}

Note: Spearman's rho; ${ }^{*} p \leq 0,01 ;{ }^{* *} p \leq 0,05$.

Source: By authors.

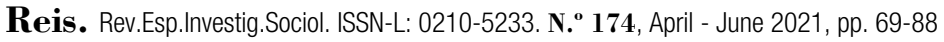


In a subsequent operation, we applied non-parametric Spearman's correlation analysis. The relationship between the independent variables (gender, age, and monthly incomes) and the answers obtained from the five-point Likert scale as the dependent variable was examined (Table 7). The results revealed a statistically significant correlation between certain of the variables and several statements: "The Balkan migration route affects the decision-making process" and gender; "New border management policy negatively influences destination choice" and age; and "The media image of the current security situation increases the sense of insecurity of potential tourists" and gender and monthly income. In all analysed cases, a small negative correlation was recorded.

\section{The impact of the political crisis on travel behaviour}

Furthermore, to determine whether the travel habits of the Serbian young adult and younger middle-aged population had changed, we also analysed travel behaviour and attitudes before and during the crisis. Most respondents (more than $70 \%$ ) confirmed that they travelled during the 2013-2015 period. Among them, two-thirds were women between 19-29 years old, who are highly educated and employed, with an average monthly income of 200-450 euros. The results showed that the Serbian young adult and younger middleaged population most often visited the following destinations: Greece (37.7\%), Montenegro (25.7\%), Turkey (9.9\%), and Croatia (8.0\%). A smaller part of the respondents spent their vacations in Italy (3.8\%), Egypt (3.3\%), Spain (3.3\%) and Tunisia (0.7\%). The results of our survey mostly coincide with the official data shown in Table 8 (Statistical Office of the Republic of Serbia, 2011-2016). The data show the number of tourists using organised packages provided by local travel agencies broken down by destination, excluding Croatia. The Statistical Office does not provide data about the number of tourists who travelled to Croatia through travel agencies because only a small number of Serbian travel agencies offer vacation packages for Croatia and most tourists organize their trips on their own, as the results of our survey confirmed once again. Additionally, and for the same reason, the total number of Serbian tourists who travel to Greece and Montenegro is presented in Table 9.

TABLE 8. The number of tourist arrivals from Serbia in 2010-2015 - major travel destinations

\begin{tabular}{lcccccc}
\hline Destination & $\mathbf{2 0 1 0}$ & $\mathbf{2 0 1 1}$ & $\mathbf{2 0 1 2}$ & $\mathbf{2 0 1 3}$ & $\mathbf{2 0 1 4}$ & $\mathbf{2 0 1 5}$ \\
\hline Greece & 213,488 & 259,283 & 261,467 & 359,894 & 368,483 & 400,575 \\
Montenegro & 66,429 & 70,180 & 73,999 & 75,117 & 65,453 & 60,992 \\
Croatia & - & - & - & - & - & - \\
Turkey & 51,565 & 56,167 & 65,863 & 66,589 & 66,692 & 62,089 \\
Egypt & 32,483 & 22,455 & 25,264 & 11,656 & 18,083 & 6,102 \\
Tunisia & 25,808 & 11,808 & 18,558 & 14,656 & 11,683 & 8,157 \\
Spain & 17,746 & 26,372 & 26,533 & 26,692 & 26,232 & 23,432 \\
Italy & 27,713 & 28,488 & 27,246 & 25,082 & 29,469 & 39,988 \\
\hline
\end{tabular}

Source: Statistical Office of the Republic of Serbia, 2011-2016. 
TABLE 9. Total number of tourist arrivals from Serbia 2010-2015

\begin{tabular}{lrrrrrc}
\hline \multicolumn{1}{c}{ Destination } & \multicolumn{1}{c}{$\mathbf{2 0 1 0}$} & \multicolumn{1}{c}{$\mathbf{2 0 1 1}$} & $\mathbf{2 0 1 2}$ & $\mathbf{2 0 1 3}$ & $\mathbf{2 0 1 4}$ & \multicolumn{1}{c}{$\mathbf{2 0 1 5}$} \\
\hline Greece & $1,097,505$ & $1,099,377$ & 620,450 & 778,765 & 985,661 & 727,831 \\
Montenegro & 314,836 & 301,094 & 337,245 & 303,135 & 287,620 & 372,912 \\
Croatia & 87,000 & 87,000 & 86,000 & 89,768 & 94,085 & 101,397 \\
\hline
\end{tabular}

Source: Croatian Bureau of Statistics, 2011-2017; Hellenic Statistical Authority, 2011-2016; Statistical Office of Montenegro, 2011-2016.

As Altindag (2014) and Law (2006) had argued that potential risk could lead to the cancellation of trips, the travel intentions for the summer holidays in the year 2016 were analysed. Approximately $63 \%$ responded affirmatively that they would travel. Among those who decided to travel, the largest proportion were women aged 19-29 (over $40 \%)$. The majority were highly educated (> $50 \%$ ), while $35 \%$ had an average monthly income of 200-450 euros. It should be noted that a significant number of the respondents had not decided at the time of the survey $(18.1 \%)$, while $18.9 \%$ did not plan to travel during summer 2016. Compared to previous years, some changes occurred regarding the destination selected in 2016. The results show that the top two destinations were still Greece (36.9\%) and Montenegro (7.6\%). Turkey had been in third place among Serbian tourists during the pre-crisis period, but it dropped to sixth place (1.2\%). Croatia (3.6\%), Spain, and Italy $(2.8 \%)$ each rose one place. The change was the most significant for North African destinations, with Egypt, and Tunisia no longer in the top-ten list of destinations among Serbian young adult and younger middle-aged tourists. In 2016, their share was less than $0.5 \%$.

The Republic of Serbia used to be part of the Socialist Federal Republic of Yugoslavia (SFRY), together with Bosnia and Herzegovina, Croatia, North Macedonia, Montenegro and Slovenia. Tourism was an important factor in social and national poli- cies and in the concept of a socialist state. With improved living standards, this social phenomenon became an important feature of the lifestyle and the identity of Yugoslav citizens. It offered a new experience of leisure and holiday making, with a specific type of consumerism through organised tours, voluntary youth labour actions, and mass property ownership (e.g., weekend cottages or "vikendica" in Serbian) (Grandits and Taylor, 2010). After the fall of the socialist regime, the Republic of Serbia remained part of the federal state until 2006, together with Montenegro. The cultural closeness (the same language, religion and history), destination proximity and the access to the Adriatic Coast are the main reasons why Serbian tourists still have a habit of spending holidays in this country. Until the 1990s, Serbian tourists frequently visited Croatia. In 1986, tourists from Serbia made more than six million overnight stays in Croatia (Kobašić, 1987), as opposed to only 477 thousand in 2016 (Croatian Bureau of Statistics, 2017). One of the reasons was the Yugoslav civil war in the 1990s, which led to the breakdown of the SFRY and resulted in tensions between the two countries. The bulk of tourists were directed towards Greece, and a much smaller part towards Serbia's southern neighbour, Montenegro. Along with these political issues, beautiful shores, a similar culture, good services and affordable prices are reasons why Greece has become a preferred destination among Serbian tourists. Even though 
the Eastern Greek islands (e.g., Samos, Lesvos) were affected by a serious migration crisis starting in 2015 and bookings for these islands dropped by up to $90 \%$ in 2016 (Angelopoulou and Roeder, 2016), Greece has remained the number one destination among Serbian young adult and younger middle-aged tourists. Despite the crisis on the Eastern Aegean islands, Serbian tourists perceive that northern parts, where they usually take holidays, are safe.

At the same time, Turkey was rising as a relatively new destination among Serbian tourists. Its share as a tourist destination grew constantly in the 2008-2014 period. However, political instability and terrorist attacks have led to a decrease in the number of Serbian tourists that visit Turkey. The most prominent decline was registered in 2015/2016 due to two terrorist attacks and a coup d'etat attempt in Ankara and Istanbul in 2016. These events deepened the political and security crisis. Additionally, the geographical position of Turkey and the proximity of Syria and Iraq increased the anxiety of potential tourists, as a result, they avoided this destination (Teoman, 2017). Tourist demand for North African countries has also decreased in recent years due to political instability and terrorist attacks, some of which targeted Western tourists (e.g., Egypt: 1997, 2004/5, 2009, 2011, 2015-2016; Tunisia: 2002, 2013, 20152016). During these years, many package trips were cancelled and the main tourist attractions were abandoned (Tomazos, 2017).

Lastly, we sought to determine to what extent political instability and terrorism in destinations influenced the tourist decision-making process. Only $30 \%$ of respondents confirmed that recent events decisively affected their travel decisions. These are mostly women from the 19-29 age group, employed (with monthly incomes of 200-450 euros) or students. These respondents explained why the terrorist attacks in Turkey, Egypt, and Tunisia and general political instability in the MENA region affected their destination choice. In the previous period, authorities broadcast warnings concerning travel to these countries. The media reported on the turbulent events in the mentioned area and following authorities' recommendations, only a small number of agencies offered travel arrangements for the listed destinations. Even those who did not plan to travel during 2016 would have avoided North African countries and Turkey. The most common opinion among those taking family vacations was:

Although there are very favourable arrangements for travelling to Egypt, Tunisia and Turkey, terrorist actions (plane crashes, attacks at beach resorts and museums), as well as the media's image of the insecurity of tourists, deterred us from travelling to these countries (ID 208).

Furthermore, some considered the following:

When it comes to choosing a destination, I would definitely not choose the countries of Islamic culture, because I think that there is a greater concentration of extremists, so I would probably feel very unsafe at all times and at every step. Why would I travel somewhere in fear, where there is a possibility that someone might blow me up? (ID 216).

Unfortunately, this opinion is in line with the conclusion made in the study by Desrues (2009: 9) that "Muslim people as a whole are perceived as Islamists". In addition, a high percentage of the respondents $(70 \%) \mathrm{em}$ phasised that the security situation did not affect their decisions because they traditionally travelled to Greece and Montenegro, which they considered safe. Some of the many similar opinions were:

I think the Greeks will do more about security because a potential terrorist attack would destroy the season and at the same time break the budget that relies heavily on tourism (ID 221).

and

Last year, during the migration crisis everything went great, so there is no reason to avoid it this year (ID 146). 
A part of the respondents believed that lesser-known destinations, which do not attract a large number of tourists should be chosen, as they were less exposed to the risk and impact of terrorism:

Essentially, the popular destinations of Western tourists should be avoided. This includes the places that were or were considered to be targets of terrorists (e.g., Egypt, Tunisia, Turkey, but also France and Italy) (ID 62).

This study also reveals that there is a small group of so-called "risk takers". They are usually less concerned about security and safety in the destination during the decision-making process (Sönmez and Graefe, 1998). Several respondents stated they were not concerned about potential terrorist attacks: "I do not care about such things at all, terrorist attacks cannot frighten me" (ID 100) or "I do not think the situation is so critical, moreover, I would use the cheap arrangements for Egypt and Tunisia" (ID 244).

This study reveals that security is an important but not decisive factor in the process of destination selection for Serbian young adult and younger middle-aged tourists. The most significant factors are price, followed by service quality and security. Regarding the limited economic power of the Serbian population, an especially researched factor in tourism demand, it can be understood why the price of travel to a tourist destination is the most significant factor (Micić, Denda and Popescu, 2019).

\section{Conclusions}

The recent security crisis in the Mediterranean region has had various consequences at both local and global levels. These are reflected in regime changes in the countries of the MENA region, migrations, the strengthening of extremist movements, terrorist attacks, and the spread of a climate of fear, all of which have certainly had an impact on the tourism industry. Altindag (2014) and Neumayer (2004) pointed out that personal safety is an important factor in destination choice, so if the perceived safety risk is high, potential tourists are likely to reconsider their destination choice. Fourie et al. (2020) argue that terrorism and crime have a negative impact on inbound tourism.

The results of our study reveal that young adult and younger middle-aged tourists from Serbia are aware of the current crisis in the MENA region. They mostly agree that the "Arab Spring" and related events negatively influence tourism in the region and that the media increase their sense of insecurity. Once again it is confirmed that the media has played a significant role during this crisis period because they transmit information about the security situation. One issue that tourism destinations are faced with is exaggeration of the situation, which can do lasting harm to their image (Ghaderi, Saboori and Khoshkam, 2017; Tomazos, 2017). The media sees terrorist attacks, political instability, wars, etc. as important news, to the point where details of questionable credibility are often disseminated (Ghaderi, Saboori and Khoshkam, 2017; Mansfeld and Pizam, 2006; Teoman, 2017).

Many studies have shown that risk perception depends on gender, age, education level, personality type, nationality and previous travel experience (Fuchs and Reichel, 2011; Lepp and Gibson, 2003; Marion, 2018; Pizam et al., 2004; Sönmez and Graefe, 1998). Despite the findings of Carr (2001), Lepp and Gibson (2003), and Sönmez and Graefe (1998), a significant difference by gender was found, indicating that gender has an impact on risk perception. In addition, a weak correlation was found between age and income and concerns about security issues.

Furthermore, the results of the research conducted among Serbian young adult and younger middle-aged tourists confirm that 
some changes in travel behaviour and the relocation of tourism demand occurred during the emergence of the migration crisis caused by the "Arab Spring". Political turmoil and terrorist attacks in Turkey and North African destinations (Egypt and Tunisia) damaged their image as destinations and increased perceived risk among tourists, so these destinations were avoided by a part of Serbia's younger adult population. It is clear that terrorism decreases inbound tourism and damages the tourism industry (Drakos and Kutan, 2003; Llorca-Vivero, 2008).

Even though several studies (Morakabati, 2007; Reisinger and Mavondo, 2005) have shown that security and safety are important factors in the travel decision process, the results of our study reveal that only a minority of the respondents tend to avoid destinations faced with security issues. Most of the respondents considered security of high but not decisive importance in destination selection and in making the decision whether to travel or not. This attitude could be explained by the political instability and wars that Serbian inhabitants have faced. The instability in their country of origin may be a factor in their higher tolerance for political instability, crime and terrorism in possible destination countries (Fourie, Roselló-Nadal and Santana-Gallego, 2010). Another factor is certainly the low purchasing power of Serbian citizens. Among those who did not plan to travel during summer 2016, they emphasized that they certainly would not have chosen destinations considered as unsafe, such as Turkey, Egypt, and Tunisia. This opinion was especially widespread among families with infants and young children. A strategy in response to this concern is travelling to less popular tourist destinations, which implies changes in tourism demand.

Since our survey was conducted among a specific segment of the Serbian population and refers to a particular crisis, the results could be generalized to similar populations. In any case, the findings of this study facilitate our understanding of the role of security and safety in making travel decisions and destination selection, specifically among the young adult and younger middle-aged population. These findings could also help policymakers understand the significance of safety and security in destination image. It is necessary to emphasize that stakeholders at all levels should work together to reduce insecurity in destinations, because of their vulnerability to security issues (Fourie, Roselló-Nadal and Santana-Gallego, 2020).

In contemporary circumstances, this type of research can be carried out in various geographic regions faced with similar issues and as a starting point for further empirical analysis both in tourism and security studies. Future research should look at other socioeconomic, ethnic, religious and personal characteristics of respondents and their attitude towards risk factors. As the study conducted by Ghaderi, Saboori and Khoshkam, (2017) suggested that physical security is just one factor in tourism destination safety, others should also be examined, such as human rights, bio-security, and environmental security. In the end, further study could be conducted to determine whether this political crisis and related events have had short term impacts or are having longer term impacts, keeping in mind that terrorist attacks usually have short term effects, while political instability can lead to disruption of destination image (Araña and León, 2008; Saha and Yap, 2014).

\section{Biblography}

Altindag, Duha T. (2014). "Crime and International Tourism". Journal of Labor Research, 35(1): 1-14. doi: 10.1007/s12122-014-9174-8

Angelopoulou, Alexia and Roeder, Bernd (2016). Greek Tourism Still Affected by Refugee Crisis. Available at: http://www.iol.co.za/travel/world/ europe/greek-tourism-still-affected-by-refugeecrisis-2003282, access June 30, 2019. 
Ansani, Andrea and Daniele, Vittorio (2012). "About a Revolution: The Economic Motivations of the Arab Spring". International Journal of Development and Conflict, 2(3): 1-25. doi: 10.1142/ S2010269012500135

Araña, Jorge E. and León, Carmelo J. (2008). "The impact of terrorism on tourism demand". Annals of Tourism Research, 35(2): 299-315. doi: 10.1016/j.annals.2007.08.003

Ardıç, Nurullah (2012). "Understanding the 'Arab Spring': Justice, Dignity, Religion and International Politics". Afro Eurasian Studies, 1(1): 8-52.

Avraham, Eli (2015). "Destination Image Repair during Crisis: Attracting Tourism during the Arab Spring Uprisings". Tourism Management, 47: 224-232. doi.org/10.1016/j. tourman.2014.10.003

Ballard, Chet and Prine, Rudy (2002). "Citizen Perceptions of Community Policing: Comparing Internet and Mail Survey Responses". Social Science Computer Review, 20(4): 485-493. doi: 10.1177/089443902237324

Butler, Richard and Suntikul, Wantanee (eds.) (2013). Tourism and War. London and New York: Routledge.

Carr, Neil (2001). "An Exploratory Study of Gendered Differences in Young Tourists Perception of Danger within London". Tourism Management, 22(5): 565-570. Available at: http://dx.doi. org/10.1016/S0261-5177(01)00014-0

Cohen, Erik (1972). "Toward a Sociology of International Tourism". Social Research, 39(1): 164-182. Available at: http://www.jstor.org/ stable/40970087, access February 17, 2020.

Costello, Matthew, J.; Jenkins, Craig J. and Aly, Hassan (2015). "Bread, Justice, or Opportunity? The Determinants of the Arab Awakening Protests". World Development, 67: 90-100. doi: 10.1016/j.worlddev.2014.10.002

Croatian Bureau of Statistics (2011-2017). Tourist Arrivals and Nights. Available at: https://www. dzs.hr/Hrv_Eng/publication/, access February 17, 2020.

Crompton, John L. (1979). "Motivations for Pleasure Vacation". Annals of Tourism Research, 6(4): 408-424. doi: 10.1016/0160-7383(79)90004-5

D'Alema, Francesco (2017). The Evolution of Turkey's Syria Policy. Available at: http://www.iai. it/sites/default/files/iaiwp1728.pdf, access May 3, 2019.
Dalmasso, Emanuela and Cavatorta, Francesco (2013). "Democracy, Civil Liberties and the Role of Religion after the Arab Awakening: Constitutional Reforms in Tunisia and Morocco". Mediterranean Politics, 18(2): 225-241. doi: 10.1080/13629395.2013.799341

Desrues, Thierry (2009). "El islamismo en el mundo árabe. Interpretaciones de algunas trayectorias políticas". Revista Internacional de Sociología, 67(1): 9-28. doi: 10.3989/ris.2009.i1.120

Dolnicar, Sara; Laesser, Christian and Matus, Katrina (2009). "Online Versus Paper: Format Effects in Tourism Surveys". Journal of Travel Research, 47(3): 295-316. doi: 10.1177/0047287508326506

Drakos, Konstantinos and Kutan, Ali M. (2003). "Regional Effects of Terrorism on Tourism in Three Mediterranean Countries". Journal of Conflict Resolution, 47(5): 621-641. doi: 10.1177/0022002703258198

Elsrud, Torun (2001). "Risk creation in traveling: Backpacker adventure narration". Annals of Tourism Research, 28(3): 597-617. Available at: http://dx.doi.org/10.1016/S0160-7383(00)00061-X

Enloe, Cynthia (2014). "Gender makes The World Go Around". In: Enloe, C. (ed.). Bananas, Beaches and Bases: Making Feminist Sense of International Politics. Berkeley, California: University of California Press, pp. 1-36.

Fletcher, John and Morakabati, Yeganeh (2008). "Tourism Activity, Terrorism and Political Instability within the Commonwealth: The Cases of Fiji and Kenya". International Journal of Tourism Research, 10(6): 537-556. doi: 10.1002/jtr.69

Fourie, Johan; Rosselló-Nadal, Jaume and Santana-Gallego, María (2020). "Fatal Attraction: How Security Threats Hurt Tourism". Journal of Travel Research, 59(2): 209-219. doi: 10.1177/0047287519826208

FRONTEX (2012-2016). Risk Analysis. Available at: http://frontex.europa.eu/publications, access April 13, 2019.

Fuchs, Galia and Reichel, Arie (2011). "An Exploratory Inquiry into Destination Risk Perceptions and Risk Reduction Strategies of First Time vs. Repeat Visitors to a Highly Volatile Destination". Tourism Management, 32(2): 266-276. doi: 10.1016/j.tourman.2010.01.012

Gibson, Heather and Yiannakis, Andrew (2002). "Tourist Roles: Needs and the Adult Life Course". 
Annals of Tourism Research, 29(2): 358-383. doi: 10.1016/S0160-7383(01)00037-8

Ghaderi, Zahed; Saboori, Behnaz and Khoshkam, Mana (2017). "Does Security Matter in Tourism Demand?". Current Issues in Tourism, 20(6): 552565. doi: 10.1080/13683500.2016.1161603

Grandits, Hannes and Taylor, Karin (eds.) (2010). Yugoslavia's Sunny Side: A History of Tourism in Socialism (1950s-1980s). Budapest: CEU Press.

Gustafson, Per E. (1998). "Gender Differences in Risk Perception: Theoretical and Methodological Perspectives". Risk Analysis, 18(6): 805-811. Available at: http://dx.doi.org/10.1111/j.15396924.1998.tb01123.x

Hawes, Douglass K. (1988). "Travel-Related Lifestyle Profiles of Older Women". Journal of Travel Research, 27(2): 22-32. doi: 10.1177/004728758802700204

Hellenic Statistical Authority (2011-2016). Arrivals of Non-Residents from Abroad, By Country of Residence. Available at: https://www.statistics.gr/en/ statistics/-/publication/STO04/2010-Q1, access February 17, 2020.

Hoffman, Michael and Jamal, Amaney (2012). "The Youth and the Arab Spring: Cohort Differences and Similarities". Middle East Law and Governance, 4(1): 168-188. doi: 10.1163/187633712X632399

Iso-Ahola, Seppo E. (1982). "Toward a Social Psychological Theory of Tourism Motivation: A Rejoinder". Annals of Tourism Research, 9(2): 256262. doi: 10.1016/0160-7383(82)90049-4

Iso-Ahola, Seppo E. (1983). "Towards a Social Psychology of Recreational Travel". Leisure Studies, 2(1): 45-56. doi: 10.1080/02614368300390041

Kobašić, Antun (1987). Turizam u Jugoslaviji -razvoj, stanje i perspective / Tourism in Yugoslavia-Development, Situation and Perspectives. Zagreb: Inormator.

Kôvári, István and Zimányi, Krisztina (2011). "Safety and Security in the Age of Global Tourism (The Changing Role and Conception of Safety and Security in Tourism)". APSTRACT: Applied Studies in Agribusiness and Commerce, 5(3-4): 59-62.

Law, Rob (2006). "The Perceived Impact of Risks on Travel Decisions". International Journal of Tourism Research, 8(4): 289-300. doi: 10.1002/ jtr.576

Lepp, Andrew and Gibson, Heather (2003). "Tourist Roles, Perceived Risk and International Tourism".
Annals of Tourism Research, 30(3): 606-624. doi: 10.1016/S0160-7383(03)00024-0

Lim, Christine (1999). "A Meta-Analytic Review of International Tourism Demand". Journal of Travel Research, 37(3): 273-284. doi: $10.1177 / 004728759903700309$

Llorca-Vivero, Rafael (2008). "Terrorism and International Tourism: New Evidence". Defence and Peace Economics, 19(2): 169-188. doi: 10.1080/10242690701453917

Mansfeld, Yoel and Pizam, Abraham (eds.) (2006). Tourism, Security and Safety - From Theory to Practice. London and New York: Routledge.

Mansfeld, Yoel and Winckler, Onn (2015). "Can This Be Spring? Assessing the Impact of the 'Arab Spring' on the Arab Tourism Industry". Tourism - An International Interdisciplinary Journal, 63(2): 205-223. Available at: https://hrcak.srce.hr/index.php?show=clanak\&id_clanak_jezik=205893\&lang=en, access February 17, 2020.

Marion, Karl (2018). "Risk and Uncertainty in Travel Decision-Making: Tourist and Destination Perspective". Journal of Travel Research, 57(1): 129146. doi: $10.1177 / 0047287516678337$

Mayo, Edvard J. and Jarvis, Lance P. (1981). The Psychology of Leisure Travel: Effective Marketing and Selling of Travel Services. Boston: CBI Publishing.

Micić Jasna; Denda, Stefan and Popescu, Marius (2019). "The Significance of the Risk Related Challenges in Tourist Destination Choice". Journal of the Geographical Institute "Jovan Cvijić" SASA, 69(1): 39-52. doi: 10.2298/IJGl1901039M

Middleton, Victor T. C.; Fyall, Alan; Morgan, Michael and Ranchhod, Ashok (2009). Marketing in Travel and Tourism. Oxford: Butterworth-Heinemann. (4th edition).

Morakabati, Yeganeh (2007). Tourism, Travel Risk and Travel Risk Perceptions: A Study of Travel Risk Perceptions and the Effects of Incidents on Tourism. Bournemouth: Bournemouth University. [Doctoral Thesis]. Available at: https://ethos. bl.uk/OrderDetails.do?uin=uk.bl.ethos. 486294 , access February 8, 2020.

Neumayer, Eric (2004). "The Impact of Political Violence on Tourism: Dynamic Cross-National Estimation". Journal of Conflict Resolution, 48(2): 259-281. doi: 10.1177/0022002703262358

Neumayer, Eric and Plümper, Thomas (2016). "Spatial Spill-Overs from Terrorism on Tourism: Wes- 
tern Victims in Islamic Destination Countries". Public Choice, 169(3): 195-206. doi: 10.1007/ s11127-016-0359-y

Niemi, Richard G.; Portney, Kent E. and King, David (2008). Sampling Young Adults: The Effects of Survey Mode and Sampling Method on Inferences about Political Behavior. Available at: http:// citation.allacademic.com/meta/p_mla_apa_research_citation/2/7/9/9/5/p279955_i ndex.html, access April 20, 2019.

Öniş, Ziya (2014). "Turkey and the Arab Revolutions: Boundaries of Regional Power Influence in a Turbulent Middle East". Mediterranean Politics, 19(2): 203-219. doi: 10.1080/13629395.2013.868392

Park, Kwangsoo and Reisinger, Yvette (2010). "Differences in the Perceived Influence of Natural Disasters and Travel Risk on International Travel". Tourism Geographies, 12(1): 1-24. doi: 10.1080/14616680903493621

Perles-Ribes, José Francisco; Ramón-Rodríguez, Ana Belén; Moreno-Izquierdo, Luis and Torregrosa Martí, María Teresa (2018). "Winners and losers in the Arab uprisings: a Mediterranean tourism perspective". Current Issues in Tourism, 21(16): 1810-1829. doi: 10.1080/13683500.2016.1225697

Pizam, Abraham; Reichel, Arie; Boemmel, Hermann van and Jeong, Gang-Hoan (2004). "The Relationship between Risk-Taking, Sensation-Seeking, and the Tourist Behavior of Young Adults: A Cross-Cultural Study". Journal of Travel Research, 42(3): 251-260. doi: 10.1177/0047287503258837

Plog, Stanley C. (1974). "Why Destination Areas Rise and Fall in Popularity". Cornell Hotel and Restaurant Quarterly, 14(4): 55-58. doi: 10.1177/001088047401400409

Reisinger, Yvette and Mavondo, Felix (2006). "Cultural Differences in Travel Risk Perception". Journal of Travel \& Tourism Marketing, 20(1): 13-31. doi: 10.1300/J073v20n01_02

Roehl, Wesley S. and Fesenmaier, Daniel R. (1992). "Risk Perceptions and Pleasure Travel: An Exploratory Analysis". Journal of Travel Research, 30(4): 17-26. doi: 10.1177/004728759203000403

Saha, Shrabani and Yap, Ghialy (2014). "The Moderation Effects of Political Instability and Terrorism on Tourism Development: A Cross-country Panel Analysis". Journal of Travel Research, 53(4): 509521. doi: $10.1177 / 0047287513496472$
Salih, Kamal Eldin Osman (2013). "The Roots and Causes of the 2011 Arab Uprisings". Arab Studies Quarterly, 35(2): 184-206. doi: 10.13169/ arabstudquar.35.2.0184

Sönmez, Sevil F. and Graefe, Alan R. (1998). "Influence of Terrorism Risk on Foreign Tourism Decisions". Annals of Tourism Research, 25(1): 112144. doi: 10.1016/S0160- 7383(97)00072-8

Statistical Office of Montenegro (2011-2016). Statistical Yearbook. Available at: http://monstat.org/ eng/publikacije_page.php?id=196, access February 17, 2020.

Statistical Office of the Republic of Serbia (20112016). Statistical Yearbooks of the Republic of Serbia. Available at: http://www.stat.gov.rs/publikacije/, access April 20, 2019.

Steiner, Christian (2007). "Political Instability, Transnational Tourist Companies and Destination Recovery in the Middle East after 9/11". Tourism and Hospitality Planning \& Development, 4(3): 169-190. doi: 10.1080/14790530701733421

Tarlow, Peter E. (2006). "A Social Theory of Terrorism and Tourism". En: Mansfred, Y. and Pizam, A. (eds.). Tourism Security and Safety - From Theory to Practice. London and New York: Routledge, pp. 33-47.

Tarlow, Peter E. (2014). Tourism Security - Strategies for Effectively Managing Travel Risk and Safety. Amsterdam: Butterworth-Heinemann.

Teoman, Denis Can (2017). "Terrorism and tourism in Europe, new partners". European Journal of Geography, 8(2): 132-142.

Theocharous, Antonis L.; Zopiatis, Anastasios; Lambertides, Neophytos; Savva, Christos S. and Mansfeld, Yoel (2018). "Tourism, Instability and Regional Interdependency: Evidence from the Eastern-Mediterranean". Defence and Peace Economics, 0(0): 1-24. doi: 10.1080/10242694.2018.1501531

Tomazos, Konstantinos (2017). "Egypt's Tourism Industry and the Arab Spring". In: Butler, R. and Suntikul, W. (eds.). Tourism and Political Change. Oxford: Goodfellow Publishers. (2nd edition).

UNWTO (2011-2017). UNWTO Tourism Highlights, 2011-2017 Editions. Available at: https://www.eunwto.org/action/showPubli cations? category $=10.1555 \% 2 \mathrm{Fcategory} .4000$ 0037 \&pageSize $=20 \&$ startPage $=2$, access May 20, 2019.

Vanhove, Norbert (2011). The Economics of Tourism Destinations. London: Elsevier. 
World Economic Forum (2011-2017). The Travel \& Tourism Competitiveness Report. Available at: http:// www.weforum.org/reports, access April 20, 2019.

Wearing, Betsy and Wearing, Stephen (1996). "Refocusing the Tourist Experience: The Flaneur and the Choraster". Leisure Studies, 15(4): 229-243.
Yılmaz, Bahri (2012). "Turkey and the Arab Spring: The Revolutions in Turkey's Near- Abroad”. In: Calleya, S. and Wohlfeld, M. (eds.). Change and Opportunities in the Emerging Mediterranean. Malta: Mediterranean Academy of Diplomatic Studies, pp. 349-369.

RECEPTION: July 18, 2019

REVIEW: January 14, 2020

ACCEPTANCE: May 13, 2020 